ESAIM: M2AN

Vol. 41, No 2, 2007, pp. 189-213

DOI: $10.1051 / \mathrm{m} 2 \mathrm{an}: 2007017$
ESAIM: Mathematical Modelling and Numerical Analysis

www.edpsciences.org/m2an

\title{
DIFFUSION MONTE CARLO METHOD: NUMERICAL ANALYSIS IN A SIMPLE CASE*
}

\author{
Mohamed El Makrini $^{1}$, Benjamin Jourdain ${ }^{1}$ And Tony Lelièvre ${ }^{1}$
}

\begin{abstract}
The Diffusion Monte Carlo method is devoted to the computation of electronic groundstate energies of molecules. In this paper, we focus on implementations of this method which consist in exploring the configuration space with a fixed number of random walkers evolving according to a stochastic differential equation discretized in time. We allow stochastic reconfigurations of the walkers to reduce the discrepancy between the weights that they carry. On a simple one-dimensional example, we prove the convergence of the method for a fixed number of reconfigurations when the number of walkers tends to $+\infty$ while the timestep tends to 0 . We confirm our theoretical rates of convergence by numerical experiments. Various resampling algorithms are investigated, both theoretically and numerically.
\end{abstract}

Mathematics Subject Classification. 81Q05, 65C35, 60K35, 35P15.

Received September 30, 2005.

\section{INTRODUCTION}

The computation of electronic structures of atoms, molecules and solids is a central problem in chemistry and physics. We focus here on electronic ground state calculations where the objective is the computation of the lowest eigenvalue (the so-called ground-state energy) $E_{0}$ of a self-adjoint Hamiltonian $H=-\frac{1}{2} \Delta+V$ with domain $D_{\mathcal{H}}(H)$ on a Hilbert space $\mathcal{H} \subset L^{2}\left(\mathbb{R}^{3 N}\right)$ where $N$ is the number of electrons (see [3] for a general introduction):

$$
E_{0}=\inf \left\{\langle\psi, H \psi\rangle, \psi \in D_{\mathcal{H}}(H),\|\psi\|=1\right\}
$$

where $\langle\cdot, \cdot\rangle$ denotes the duality bracket on $L^{2}\left(\mathbb{R}^{3 N}\right)$ and $\|\cdot\|$ the $L^{2}\left(\mathbb{R}^{3 N}\right)$-norm. For simplicity, we omit the spin variables. The function $V$ describes the interaction between the electrons, and between the electrons and the nuclei, which are supposed to be fixed point-like particles. The functions $\psi$ are square integrable, their normalized square modulus $|\psi|^{2}$ being interpreted as the probability density of the particles positions in space, and they satisfy an antisymmetry condition with respect to the numbering of the electrons, due to the fermionic nature of the electrons (Pauli principle): $\mathcal{H}=\bigwedge_{i=1}^{N} L^{2}\left(\mathbb{R}^{3}\right)$. We suppose that the potential $V$ is such that $E_{0}$ is

\footnotetext{
Keywords and phrases. Diffusion Monte Carlo method, interacting particle systems, ground state, Schrödinger operator, FeynmanKac formula.

* We thank Eric Cancès (CERMICS) and Mathias Rousset (Université Paul Sabatier, Toulouse) for many fruitful discussions and Michel Caffarel (IRSAMC, Toulouse) for introducing us to the DMC method and suggesting the toy model studied in this paper. We also thank the referees for useful suggestions which helped us to improve the first draft of this paper.

1 ENPC-CERMICS, 6-8 avenue Blaise Pascal, Cité Descartes, Champs sur Marne, 77455 Marne-la-Vallée Cedex 2, France.

makrimo@cermics.enpc.fr; jourdain@cermics.enpc.fr; lelievre@cermics.enpc.fr 
an isolated eigenvalue of $H$ (see [4] for sufficient conditions), and we denote by $\psi_{0}$ a normalized eigenfunction associated with $E_{0}$.

Due to the high dimensionality of the problem, stochastic methods are particularly well suited to compute $E_{0}$. The first particle approximation scheme of such spectral quantities was introduced in [12] for finite state space models. Convergence analysis for such interacting particle systems (both continuous or discrete in time) first appeared in [7-10]. The Diffusion Monte Carlo (DMC) method is widely used in chemistry (see $[2,17])$, but has been only recently considered from a mathematical viewpoint (see $[4,14]$ ). This method gives an estimate of $E_{0}$ in terms of the long-time limit of the expectation of a functional of a drift-diffusion process with a source term. It requires an importance sampling function $\psi_{I}$ which approximates the ground-state $\psi_{0}$ of $H$. Let us define the drift function $\boldsymbol{b}=\nabla \ln \left|\psi_{I}\right|$, the so-called local energy $E_{L}=\frac{H \psi_{I}}{\psi_{I}}$ and the DMC energy:

$$
E_{\mathrm{DMC}}(t)=\frac{\mathbb{E}\left(E_{L}\left(\boldsymbol{X}_{t}\right) \exp \left(-\int_{0}^{t} E_{L}\left(\boldsymbol{X}_{s}\right) \mathrm{d} s\right)\right)}{\mathbb{E}\left(\exp \left(-\int_{0}^{t} E_{L}\left(\boldsymbol{X}_{s}\right) \mathrm{d} s\right)\right)},
$$

where the $3 N$-dimensional process $\boldsymbol{X}_{t}$ satisfies the stochastic differential equation:

$$
\left\{\begin{array}{l}
\boldsymbol{X}_{t}=\boldsymbol{X}_{0}+\int_{0}^{t} \boldsymbol{b}\left(\boldsymbol{X}_{s}\right) \mathrm{d} s+\boldsymbol{W}_{t}, \\
\boldsymbol{X}_{0} \sim\left|\psi_{I}\right|^{2}(\boldsymbol{x}) \mathrm{d} \boldsymbol{x} .
\end{array}\right.
$$

The stochastic process $\left(\boldsymbol{W}_{t}\right)_{t \geq 0}$ is a standard $3 N$-dimensional Brownian motion. One can then show that (see [4])

$$
\lim _{t \rightarrow \infty} E_{\mathrm{DMC}}(t)=E_{\mathrm{DMC}, 0}
$$

where

$$
E_{\mathrm{DMC}, 0}=\inf \left\{\langle\psi, H \psi\rangle, \psi \in D_{\mathcal{H}}(H),\|\psi\|=1, \psi=0 \text { on } \psi_{I}^{-1}(0)\right\}
$$

We have proved in [4] that $E_{\mathrm{DMC}, 0} \geq E_{0}$, with equality if and only if the nodal surfaces of $\psi_{I}$ coincide with those of a ground state $\psi_{0}$ of $H$. In other words, if there exists a ground state $\psi_{0}$ such that $\psi_{I}^{-1}(0)=\psi_{0}^{-1}(0)$, then $\lim _{t \rightarrow \infty} E_{\mathrm{DMC}}(t)=E_{0}$. The error $\left|E_{0}-E_{\mathrm{DMC}, 0}\right|$ is related to the so-called fixed-node approximation, which is well known by practitioners of the field (see [3]).

In this paper, we complement the theoretical results obtained in [4] with a numerical analysis in a simple case. In practice, the longtime limit $E_{\mathrm{DMC}, 0}$ in (4) is approximated by taking the value of $E_{\mathrm{DMC}}$ at a (large) time $T>0$. Then $E_{\mathrm{DMC}}(T)$ is approximated by using a discretization in time of the stochastic differential equation (3) and of the integral in the exponential factor in (2), and an approximation of the expectation values in (2) by an empirical mean over a large number $N$ of trajectories. These trajectories $\left(\boldsymbol{X}^{i}\right)_{1 \leq i \leq N}$, also called walkers in the physical literature or particles in the mathematical literature, satisfy a discretized version of (3), and interact at times $n \Delta t$ for $n \in\{1, \ldots, \nu-1\}$ where $\Delta t=T / \nu$ for $\nu \in \mathbb{N}^{*}$ through a stochastic reconfiguration step aimed at reducing the discrepancy between their exponential weights. We thus obtain an interacting particle system. The number of reconfiguration steps is $\nu-1$. The stochastic differential equation (3) is discretized with a possibly smaller timestep $\delta t=\Delta t / \kappa=T /(\nu \kappa)$ with $\kappa \in \mathbb{N}^{*}$. The total number of steps for the discretization of (3) is then $K=\nu \kappa$. 
In the following, we consider the following simple version of a DMC scheme with a fixed number of walkers (see [2]):

- Initialization of an ensemble of $N$ walkers $\left(\boldsymbol{X}_{0 \Delta t}^{j}\right)_{1 \leq j \leq N}$ i.i.d. according to $\left|\psi_{I}\right|^{2}(\boldsymbol{x}) \mathrm{d} \boldsymbol{x}$.

- Iterations in time: let us be given the particle positions $\left(\boldsymbol{X}_{n \Delta t}^{j}\right)_{1 \leq j \leq N}$ at time $n \Delta t$, for $n \in\{0, \ldots, \nu-$ 1\}. The new particle positions at time $(n+1) \Delta t$ are obtained in two steps:

(1) Walkers displacement: for all $1 \leq j \leq N$, the successive positions $\left(\boldsymbol{X}_{n \Delta t+\delta t}^{j}, \ldots, \boldsymbol{X}_{n \Delta t+\kappa \delta t}^{j}\right)$ over the time interval $(n \Delta t,(n+1) \Delta t)$ are obtained by an appropriate discretization of $(3)$. In the field of interacting particles system for Feynman-Kac formulae (see [7,9]), this step is called the mutation step.

(2) Stochastic reconfiguration: The new positions ${ }^{1}\left(\boldsymbol{X}_{(n+1) \Delta t}^{j}\right)_{1 \leq j \leq N}$ which will be used as the initial particle positions on the time interval $((n+1) \Delta t,(n+2) \Delta t)$ are obtained from independent sampling of the measure

$$
\frac{\sum_{j=1}^{N} \exp \left(-\delta t \sum_{k=1}^{\kappa} E_{L}\left(\boldsymbol{X}_{n \Delta t+k \delta t}^{j}\right)\right) \delta_{\boldsymbol{X}_{n \Delta t+\kappa \delta t}^{j}}}{\sum_{j=1}^{N} \exp \left(-\delta t \sum_{k=1}^{\kappa} E_{L}\left(\boldsymbol{X}_{n \Delta t+k \delta t}^{j}\right)\right)}
$$

In words, the new particle positions $\left(\boldsymbol{X}_{(n+1) \Delta t}^{j}\right)_{1 \leq j \leq N}$ are randomly chosen among the final particle positions $\left(\boldsymbol{X}_{n \Delta t+\kappa \delta t}^{j}\right)_{1 \leq j \leq N}$, each of them being weighted with the coefficient $\exp \left(-\delta t \sum_{k=1}^{\kappa} E_{L}\left(\boldsymbol{X}_{n \Delta t+k \delta t}^{j}\right)\right)$ (accordingly to the exponential factor in $\left.(2)\right)$. In the field of interacting particles system for Feynman-Kac formulae, this step is called the selection step.

An estimate of $E_{\mathrm{DMC}}\left(t_{n+1}\right)$ is then given by:

$$
E_{\mathrm{DMC}}\left(t_{n+1}\right) \simeq \frac{1}{N} \sum_{j=1}^{N} E_{L}\left(\boldsymbol{X}_{(n+1) \Delta t}^{j}\right)
$$

There are other possible estimations of $E_{\mathrm{DMC}}\left(t_{n+1}\right)$. In [2], the authors propose to use Cesaro or weighted Cesaro means of the expression (7). In Section 1, we will use the following expression:

$$
E_{\mathrm{DMC}}\left(t_{n+1}\right) \simeq \frac{\sum_{j=1}^{N} E_{L}\left(\boldsymbol{X}_{n \Delta t+\kappa \delta t}^{j}\right) \exp \left(-\delta t \sum_{k=1}^{\kappa} E_{L}\left(\boldsymbol{X}_{n \Delta t+k \delta t}^{j}\right)\right)}{\sum_{j=1}^{N} \exp \left(-\delta t \sum_{k=1}^{\kappa} E_{L}\left(\boldsymbol{X}_{n \Delta t+k \delta t}^{j}\right)\right)},
$$

in an intermediate step to prove the convergence result.

We would like to mention that a continuous in time version of the DMC scheme with stochastic reconfiguration has been proposed in [14]. The author analyzes the longtime behavior of the interacting particle system and proves in particular a uniform in time control of the variance of the estimated energy.

The DMC algorithm presented above is prototypical. Many refinements are used in practice. For example, an acception-rejection step is generally used in the walkers displacement step (see [13]). This will not be

\footnotetext{
${ }^{1}$ With a slight abuse of notation and though $n \Delta t+\kappa \delta t=(n+1) \Delta t$, we distinguish between the particle positions $\boldsymbol{X}_{n \Delta t+\kappa \delta t}^{j}$ at the end of the walkers displacement on time interval $(n \Delta t,(n+1) \Delta t)$, and the new particle positions $\boldsymbol{X}_{(n+1) \Delta t}^{j}$ obtained after the reconfiguration step, and which are used as the initial position for the next walkers displacement on time interval $((n+1) \Delta t,(n+2) \Delta t)$. We will use a more precise notation for the analysis of the numerical scheme in Section 1 , but this is not required at this stage.
} 
discussed here. Likewise, the selection step can be done in many ways (see [5,6] for general algorithms, and [2, 15,17 for algorithms used in the context of DMC computations). In this paper, we restrict ourselves to resampling methods with a fixed number of particles, and such that the weights of the particles after resampling are equal to 1 . Then, the basic consistency requirement of the selection step is that, conditionally on the former positions $\left(\boldsymbol{X}_{n \Delta t+k \delta t}^{j}\right)_{1 \leq j \leq N, 1 \leq k \leq \kappa}$, the $i$-th particle $\boldsymbol{X}_{n \Delta t+\kappa \delta t}^{i}$ is replicated $N \rho_{n}^{i}$ times in mean, where $\rho_{n}^{i}=\exp \left(-\delta t \sum_{k=1}^{\kappa} E_{L}\left(\boldsymbol{X}_{n \Delta t+k \delta t}^{i}\right)\right) / \sum_{j=1}^{N} \exp \left(-\delta t \sum_{k=1}^{\kappa} E_{L}\left(\boldsymbol{X}_{n \Delta t+k \delta t}^{j}\right)\right)$ denotes the (normalized) weight of the $i$-th particle. There are of course many ways to satisfy this requirement. We presented above the so-called multinomial resampling method. We will also discuss below residual resampling (also called stochastic remainder resampling), stratified resampling and systematic resampling, which may also be used for DMC computations. Let us briefly describe these three resampling methods. Residual resampling consists in reproducing $\left\lfloor N \rho_{n}^{i}\right\rfloor$ times the $i$-th particle, and then completing the set of particles by using multinomial resampling to draw the $N^{R}=N-\sum_{l=1}^{N}\left\lfloor N \rho_{n}^{l}\right\rfloor$ remaining particles, the $i$-th particle being assigned the weight $\rho_{n}^{R, i}=\left\{N \rho_{n}^{i}\right\} / N^{R}$. Here and in the following, $\lfloor x\rfloor$ and $\{x\}$ respectively denote the integer and the fractional part of $x \in \mathbb{R}$. In the stratified resampling method, the interval $(0,1)$ is divided into $N$ intervals $((i-1) / N, i / N)(1 \leq i \leq N), N$ random variables are then drawn independently and uniformly in each interval, and the new particle positions are then obtained by the inversion method: $\boldsymbol{X}_{(n+1) \Delta t}^{i}=\sum_{j=1}^{N} 1_{\left\{\sum_{l=1}^{j-1} \rho_{n}^{l}<\left(i-U_{n}^{i}\right) / N \leq \sum_{l=1}^{j} \rho_{n}^{l}\right\}} \boldsymbol{X}_{n \delta t+\kappa \delta t}^{j}$, where $U_{n}^{i}$ are i.i.d. random variables uniformly distributed over [0,1]. Here and in the following, we use the convention $\sum_{l=1}^{0} \cdot=0$. Systematic resampling consists in replicating the $i$-th particle $\left\lfloor N \sum_{l=1}^{i} \rho_{n}^{l}+U_{n}\right\rfloor-\left\lfloor N \sum_{l=1}^{i-1} \rho_{n}^{l}+\right.$ $\left.U_{n}\right\rfloor$ times $^{2}$, where $\left(U_{n}\right)_{n \geq 1}$ are independent random variables uniformly distributed in $[0,1]$. Notice that systematic resampling can be seen as the stratified resampling method, with $U_{n}^{1}=\ldots=U_{n}^{N}=U_{n}$. Contrary to the three other resampling methods, after a systematic resampling step, the new particle positions are not independent, conditionally on the former positions. This makes systematic resampling much more difficult to study mathematically. To our knowledge, its convergence even in a discrete time setting is still an open question. We will therefore restrict ourselves to a numerical study of its performance.

Notice that practitioners often use branching algorithms with an evolving number of walkers during the computation (see $[13,17])$ : the particles with low local energy are replicated and the particles with high local energy are killed, without keeping the total number of particles constant. This may lead to a smaller Monte Carlo error (fourth contribution to the error in the classification just below).

We can distinguish between four sources of errors in the approximation of $E_{0}$ by $\frac{1}{N} \sum_{j=1}^{N} E_{L}\left(\boldsymbol{X}_{\nu \Delta t}^{j}\right)$ :

(1) the error due to the fixed node approximation $\left|E_{0}-E_{\mathrm{DMC}, 0}\right|$;

(2) the error due to finite time approximation of the $\operatorname{limit}_{\lim } \lim _{t \rightarrow \infty} E_{\mathrm{DMC}}(t) \simeq E_{\mathrm{DMC}}(T)$;

(3) the error due to the time discretization of the stochastic differential equation (3) and of the integral in the exponential factor in $E_{\mathrm{DMC}}(t)$ (see $\left.(2)\right)$;

(4) the error introduced by the interacting particle system, due to the approximation of the expectation value in (2) by an empirical mean.

The error (1) due to the fixed node approximation has been analyzed theoretically in [4].

Concerning the error (2) due to finite time approximation of the limit, the rate of convergence in time is typically exponential. Indeed if $H$ admits a spectral gap (namely if the distance between $E_{0}$ and the remaining of the spectrum of $H$ is strictly positive), and if $\psi_{I}$ is such that $\left\langle\psi_{I}, H \psi_{I}\right\rangle<\inf \sigma_{\text {ess }}(H)$, then one can show that the operator $H$ with domain $D_{\mathcal{H}}(H) \cap\left\{\psi, \psi=0\right.$ on $\left.\psi_{I}^{-1}(0)\right\}$ (whose lowest eigenvalue is $E_{\mathrm{DMC}, 0}$, see $(5)$ )

\footnotetext{
${ }^{2}$ The consistency of this resampling method follows from the following easy computation

$$
\mathbb{E}(\lfloor x+U\rfloor)=\lfloor x\rfloor \mathbb{P}(U<1-\{x\})+(\lfloor x\rfloor+1) \mathbb{P}(U \geq 1-\{x\})=\lfloor x\rfloor(1-\{x\})+(\lfloor x\rfloor+1)\{x\}=x .
$$
}


also admits a spectral gap $\gamma>0$. Then, by standard spectral decomposition methods, we have:

$$
0 \leq\left|E_{\mathrm{DMC}}(t)-E_{\mathrm{DMC}, 0}\right| \leq C \exp (-\gamma t)
$$

Our aim in this paper is to provide some theoretical and numerical results related to the errors (3) and (4), in the framework of a simple one-dimensional case. We therefore consider in the following that the final time of simulation $T$ is fixed and we analyze the error introduced by the numerical scheme on the estimate of $E_{\mathrm{DMC}}(T)$. Our convergence result is of the form:

$$
\mathbb{E}\left|E_{\mathrm{DMC}}(T)-\frac{1}{N} \sum_{j=1}^{N} E_{L}\left(\boldsymbol{X}_{\nu \kappa \delta t}^{j}\right)\right| \leq C(T) \delta t+\frac{C(T, \nu)}{\sqrt{N}},
$$

where $C(T)$ (resp. $C(T, \nu)$ ) denotes a constant which only depends on $T$ (resp. on $T$ and $\nu$ ) (see Thm. 4 and Cor. 13 below).

Let us now present the toy model we consider in the following. We consider the Hamiltonian

$$
H=-\frac{1}{2} \frac{\mathrm{d}^{2}}{\mathrm{~d} x^{2}}+V, \text { with } V=\frac{\omega^{2}}{2} x^{2}+\theta x^{4}
$$

where $\omega, \theta>0$ are two constants. The ground state energy $E_{0}$ is defined by (1), with

$$
\mathcal{H}=\left\{\psi \in L^{2}(\mathbb{R}), \psi(x)=-\psi(-x)\right\}
$$

We restrict the functional spaces to odd functions in order to mimic the antisymmetry constraint on $\psi$ for fermionic systems. The importance sampling $\psi_{I}$ is chosen to be the ground state of $H_{0}=-\frac{1}{2} \frac{\mathrm{d}^{2}}{\mathrm{~d} x^{2}}+\frac{\omega^{2}}{2} x^{2}$ on $\mathcal{H}$ :

$$
\psi_{I}(x)=\sqrt{2 \omega}\left(\frac{\omega}{\pi}\right)^{1 / 4} x \mathrm{e}^{-\frac{\omega}{2} x^{2}} .
$$

It is associated with the energy $\frac{3}{2} \omega: H_{0} \psi_{I}=\frac{3}{2} \omega \psi_{I}$. The drift function $b$ and the local energy $E_{L}$ are then defined by:

$$
b(x)=\frac{\psi_{I}^{\prime}}{\psi_{I}}(x)=\frac{1}{x}-\omega x, \text { and } E_{L}(x)=V(x)-\frac{1}{2} \frac{\psi_{I}^{\prime \prime}}{\psi_{I}}(x)=\frac{3}{2} \omega+\theta x^{4} .
$$

Thus, using equation (2), the DMC energy is:

$$
E_{\mathrm{DMC}}(t)=\frac{3}{2} \omega+\theta \frac{\mathbb{E}\left(X_{t}^{4} \exp \left(-\theta \int_{0}^{t} X_{s}^{4} \mathrm{~d} s\right)\right)}{\mathbb{E}\left(\exp \left(-\theta \int_{0}^{t} X_{s}^{4} \mathrm{~d} s\right)\right)}
$$

where

$$
X_{t}=X_{0}+\int_{0}^{t}\left(\frac{1}{X_{s}}-\omega X_{s}\right) \mathrm{d} s+W_{t}
$$

with $\left(W_{t}\right)_{t \geq 0}$ a Brownian motion independent from the initial variable $X_{0}$ which is distributed according to the invariant measure $2 \psi_{I}^{2}(x) 1_{\{x>0\}} \mathrm{d} x$. We recall that due to the explosive part in the drift function $b$, the stochastic process cannot cross 0 , which is the zero point of $\psi_{I}$ (see [4]): $\mathbb{P}\left(\exists t>0, X_{t}=0\right)=0$. This explains why the restriction of $\psi_{I}^{2}$ to $\mathbb{R}_{+}^{*}$ is indeed an invariant measure for (15). For $\theta>0$, the longtime limit $E_{\mathrm{DMC}, 0}$ of $E_{\mathrm{DMC}}(t)$ is not analytically known, but can be very accurately computed by a spectral method (see Sect. 2.1). Let us finally make precise that for the numerical analysis, we use a special feature of our simple model, namely the fact that for $s \leq t$, it is possible to simulate the conditional law of $X_{t}$ given $X_{s}$ (see Appendix). The time discretization error is thus only related to the discretization of the integral in the exponential factor in the DMC 
energy (2). We however indicate some possible ways to prove (1) with a convenient time discretization of the SDE (see Eq. (17), Rem. 3 and Prop. 14).

Though our model is one-dimensional (and therefore still far from the real problem (1)), it contains one of the main difficulties related to the approximation of the ground state energy for fermionic systems, namely the explosive behavior of the drift in the stochastic differential equation. However, two characteristics of practical problems are missing in the toy model considered here. First, since we consider a one-particle model, we do not treat difficulties related to singularities of the drift and of the local energy at points where two particles (either two electrons or one electron and one nucleus) coincide. Second, the local energy $E_{L}$ generally explodes at the nodes of the trial wave function, and this is not the case on the simple example we study since the trial wave function is closely related to the exact ground state. For an adaptation of the DMC algorithm to take care of these singularities, we refer to [17]. Despite the simplicity of the model studied in this paper, we think that the convergence results we obtain and the mathematical tools we use are prototypical for generalization to more complicated systems.

Compared to previous mathematical analysis of convergence for interacting particle systems with stochastic reconfiguration [7-10,14], our study concentrates on the limit $\delta t \rightarrow 0$ and $N \rightarrow \infty$ for a fixed time $T$, and on the influence of the time discretization error in the estimate (9), where the test function $E_{L}$ is unbounded. It is actually important in our analysis that this unbounded function $E_{L}$ also appears in the weights of the particles, since it allows for specific estimates (see Lems. 9 and 11 below).

The paper is organized as follows. In Section 1, we prove the convergence result, by adapting the methods of $[7,9]$ to analyze the dependence of the error on $\delta t$. We then check the optimality of this theoretical result by numerical experiments in Section 2, where we also analyze numerically the dependence of the results on various numerical parameters, including the number $(\nu-1)$ of reconfiguration steps. From these numerical experiments, we propose a simple heuristic method to choose the optimal number of reconfiguration steps.

Notation: For any set of random variables $\left(Y_{i}\right)_{i \in I}$, we denote by $\sigma\left(\left(Y_{i}\right)_{i \in I}\right)$ the sigma-field generated by these random variables. The parameters $\omega$ and $\theta$ are fixed positive constants. By convention, any sum from one to zero is equal to zero: $\sum_{k=1}^{0} \cdot=0$. Likewise, the subset $\{1,2, \ldots, 0\}$ of $\mathbb{N}$ is by convention the empty set. For any real $x,\lfloor x\rfloor$ and $\{x\}$ respectively denote the integer and the fractional part of $x$.

\section{Numerical ANAlysis in a Simple CASE}

We perform the numerical analysis in two steps: time discretization and then particle approximation.

\subsection{Time discretization}

We recall that $T>0$ denotes the final simulation time, and that $\delta t=\frac{T}{K}$ is the smallest time-step. Since $Y_{t}=X_{t}^{2}$ is a square root process solving $\mathrm{d} Y_{t}=\left(3-2 \omega Y_{t}\right) \mathrm{d} t+2 \sqrt{Y_{t}} \mathrm{~d} W_{t}$, it is possible to simulate the increments $Y_{(k+1) \delta t}-Y_{k \delta t}$ and therefore $X_{(k+1) \delta t}-X_{k \delta t}$ (see Appendix or [11], p. 120). We can thus simulate exactly in law the vector $\left(X_{0}, X_{\delta t}, \ldots, X_{K \delta t}\right)$. That is why we are first going to study the error related to the time discretization of the integral which appears in the exponential factors in (14).

Let us define the corresponding approximation of $E_{\mathrm{DMC}}(T)$ :

$$
E_{\mathrm{DMC}}^{\delta t}(T)=\frac{\mathbb{E}\left(E_{L}\left(X_{T}\right) \exp \left(-\delta t \sum_{k=1}^{K} E_{L}\left(X_{k \delta t}\right)\right)\right)}{\mathbb{E}\left(\exp \left(-\delta t \sum_{k=1}^{K} E_{L}\left(X_{k \delta t}\right)\right)\right)}=\frac{3}{2} \omega+\theta \frac{\mathbb{E}\left(X_{T}^{4} \exp \left(-\theta \delta t \sum_{k=1}^{K} X_{k \delta t}^{4}\right)\right)}{\mathbb{E}\left(\exp \left(-\theta \delta t \sum_{k=1}^{K} X_{k \delta t}^{4}\right)\right)} .
$$

\section{Proposition 1.}

$$
\forall K \in \mathbb{N}^{*},\left|E_{\mathrm{DMC}}(T)-E_{\mathrm{DMC}}^{\delta t}(T)\right| \leq C_{T} \delta t
$$


Proof. Using Hölder inequality, we have:

$$
\begin{aligned}
\left|E_{\mathrm{DMC}}(T)-E_{\mathrm{DMC}}^{\delta t}(T)\right| \leq & \frac{\theta}{\mathbb{E}\left(\exp \left(-\theta \delta t \sum_{k=1}^{K} X_{k \delta t}^{4}\right)\right)}\left(\sqrt{\mathbb{E}\left(X_{T}^{8}\right)}+\frac{\mathbb{E}\left(X_{T}^{4} \exp \left(-\theta \int_{0}^{T} X_{s}^{4} \mathrm{~d} s\right)\right)}{\mathbb{E}\left(\exp \left(-\theta \int_{0}^{T} X_{s}^{4} \mathrm{~d} s\right)\right)}\right) \\
& \left(\mathbb{E}\left(\left(\exp \left(-\theta \int_{0}^{T} X_{s}^{4} \mathrm{~d} s\right)-\exp \left(-\theta \delta t \sum_{k=1}^{K} X_{k \delta t}^{4}\right)\right)^{2}\right)\right)^{1 / 2} .
\end{aligned}
$$

The conclusion is now a consequence of Lemma 2 and the fact that the function $x \in \mathbb{R}_{+} \rightarrow \mathrm{e}^{-\theta x}$ is Lipschitz continuous with constant $\theta$.

Lemma 2. For any $K \in \mathbb{N}^{*}$,

$$
\mathbb{E}\left(\left(\int_{0}^{T} X_{s}^{4} \mathrm{~d} s-\delta t \sum_{k=1}^{K} X_{k \delta t}^{4}\right)^{2}\right) \leq C \delta t^{2}\left(T^{2}+T\right),
$$

where $\delta t=\frac{T}{K}$.

Proof. By Itô's formula, $\mathrm{d} X_{t}^{4}=\left(10 X_{t}^{2}-4 \omega X_{t}^{4}\right) \mathrm{d} t+4 X_{t}^{3} \mathrm{~d} W_{t}$. With the integration by parts formula, one deduces that for any $k \in\{1, \ldots, K\}$,

$$
\int_{(k-1) \delta t}^{k \delta t}\left(X_{k \delta t}^{4}-X_{s}^{4}\right) \mathrm{d} s=\int_{(k-1) \delta t}^{k \delta t}(s-(k-1) \delta t)\left(\left(10 X_{s}^{2}-4 \omega X_{s}^{4}\right) \mathrm{d} s+4 X_{s}^{3} \mathrm{~d} W_{s}\right) .
$$

Therefore denoting $\tau_{s}=\left\lfloor\frac{s}{\delta t}\right\rfloor \delta t$ the discretization time just before $s$, one obtains

$$
\delta t \sum_{k=1}^{K} X_{k \delta t}^{4}-\int_{0}^{T} X_{s}^{4} \mathrm{~d} s=\int_{0}^{T}\left(s-\tau_{s}\right)\left(10 X_{s}^{2}-4 \omega X_{s}^{4}\right) \mathrm{d} s+\int_{0}^{T}\left(s-\tau_{s}\right) 4 X_{s}^{3} \mathrm{~d} W_{s} .
$$

Hence

$$
\left.\mathbb{E}\left(\left(\delta t \sum_{k=1}^{K} X_{k \delta t}^{4}-\int_{0}^{T} X_{s}^{4} \mathrm{~d} s\right)^{2}\right) \leq 2 \int_{0}^{T}\left(s-\tau_{s}\right)^{2} \mathbb{E}\left(T\left(10 X_{s}^{2}-4 \omega X_{s}^{4}\right)^{2}+16 X_{s}^{6}\right)\right) \mathrm{d} s
$$

Since $X_{0}$ is distributed according to the invariant measure $2 \psi_{I}^{2}(x) 1_{\{x>0\}} \mathrm{d} x$, so is $X_{s}$. As a consequence, for any $p \in \mathbb{N}, \mathbb{E}\left(X_{s}^{p}\right)$ does not depend on $s$ and is finite and the conclusion follows readily.

In realistic situations, exact simulation of the increments $X_{(k+1) \delta t}-X_{k \delta t}$ is not possible and one has to resort to discretization schemes. The singularity of the drift coefficient prevents the process $X_{t}$ from crossing the nodal surfaces of the importance sampling function $\psi_{I}$. The standard explicit Euler scheme does not preserve this 
property at the discretized level. For that purpose, we suggest to use the following explicit scheme proposed by [1]

$$
\left\{\begin{array}{l}
\bar{X}_{0}=X_{0} \\
\forall k \in \mathbb{N}, \bar{X}_{(k+1) \delta t}=\left(\left(\bar{X}_{k \delta t}(1-\omega \delta t)+\frac{\Delta W_{k+1}}{1-\omega \delta t}\right)^{2}+2 \delta t\right)^{1 / 2} \text { with } \Delta W_{k+1}=W_{(k+1) \delta t}-W_{k \delta t}
\end{array}\right.
$$

Because of the singularity at the origin of the drift coefficient in (15), we have not been able so far to prove the following weak error bound (see Rem. 3 below):

$$
\left|E\left(f\left(X_{T}^{4}\right) \exp \left(-\theta \int_{0}^{T} X_{s}^{4} \mathrm{~d} s\right)\right)-\mathbb{E}\left(f\left(\bar{X}_{T}^{4}\right) \exp \left(-\theta \delta t \sum_{k=1}^{K} \bar{X}_{k \delta t}^{4}\right)\right)\right| \leq C_{T} \delta t \text { for } f(x) \equiv 1 \text { and } x^{4} .
$$

Such a bound is expected according to [16] and would imply that

$$
\left|E_{\mathrm{DMC}}(T)-\frac{\mathbb{E}\left(E_{L}\left(\bar{X}_{T}\right) \exp \left(-\delta t \sum_{k=1}^{K} E_{L}\left(\bar{X}_{k \delta t}\right)\right)\right)}{\mathbb{E}\left(\exp \left(-\delta t \sum_{k=1}^{K} E_{L}\left(\bar{X}_{k \delta t}\right)\right)\right)}\right| \leq C_{T} \delta t
$$

Remark 3. We would like to sketch a possible way to prove (18). Because the square root in (17) makes expansions with respect to $\delta t$ and $\Delta W_{k+1}$ complicated, it is easier to work with $Y_{t}=X_{t}^{2}$ and $\bar{Y}_{k \delta t}=\bar{X}_{k \delta t}^{2}$ which satisfy

$$
\mathrm{d} Y_{t}=\left(3-2 \omega Y_{t}\right) \mathrm{d} t+2 \sqrt{Y_{t}} \mathrm{~d} W_{t} \text { and } \bar{Y}_{(k+1) \delta t}=\left(\sqrt{\bar{Y}_{k \delta t}}(1-\omega \delta t)+\frac{\Delta W_{k+1}}{1-\omega \delta t}\right)^{2}+2 \delta t
$$

The standard approach to analyze the time discretization error of the numerator and denominator of the left hand side of (19) is then to introduce some functions $v$ and $w$ solutions to the partial differential equation:

$$
\partial_{t} v=(3-2 y) \partial_{y} v+2 y \partial_{y y} v-\theta y^{2} v,(t, y) \in \mathbb{R}_{+} \times(0,+\infty)
$$

with initial conditions $v(0, y)=y^{2}$ and $w(0, y)=1$. Now, we write (for the numerator, for example):

$$
\begin{aligned}
& \mathbb{E}\left(X_{T}^{4} \exp \left(-\theta \int_{0}^{T} X_{s}^{4} \mathrm{~d} s\right)\right)-\mathbb{E}\left(\bar{X}_{T}^{4} \exp \left(-\theta \delta t \sum_{k=1}^{K} \bar{X}_{k \delta t}^{4}\right)\right) \\
& =\sum_{k=0}^{K-1} \mathbb{E}\left(\left(v\left(T-k \delta t, \bar{Y}_{k \delta t}\right)-\mathrm{e}^{-\theta \delta t \bar{Y}_{(k+1) \delta t}^{2}} v\left(T-(k+1) \delta t, \bar{Y}_{(k+1) \delta t}\right)\right) \exp \left(-\theta \delta t \sum_{j=0}^{k-1} \bar{Y}_{j \delta t}^{2}\right)\right) .
\end{aligned}
$$

An error bound of the form $C_{T} \delta t$ can now be proved by some Taylor expansions as in $[1,16]$, provided the existence of a sufficiently smooth solution $v$ to (20). We have not been able to prove existence of such a solution so far.

\subsection{Particle approximation}

We now introduce some notation to study the particle approximation. We recall that $\nu$ denotes the number of large timesteps (the number of reconfiguration steps is $\nu-1$ ), and $\Delta t=\kappa \delta t$ the time period between two reconfiguration steps. Let us suppose that we know the initial positions $\left(X_{n, 0}^{i}\right)_{1 \leq i \leq N}$ of the $N$ walkers at time $(n-1) \Delta t$, for a time index $n \in\{1, \ldots, \nu\}$. The successive positions of the walkers over the time interval $((n-1) \Delta t, n \Delta t)$ are then given by $\left(X_{n, \delta t}^{i}, \ldots, X_{n, \kappa \delta t}^{i}\right)$, where $\left(X_{n, t}^{i}\right)_{0 \leq t \leq \Delta t}$ satisfies:

$$
X_{n, t}^{i}=X_{n, 0}^{i}+\int_{0}^{t} b\left(X_{n, s}^{i}\right) \mathrm{d} s+\left(W_{t+(n-1) \Delta t}^{i}-W_{(n-1) \Delta t}^{i}\right) .
$$




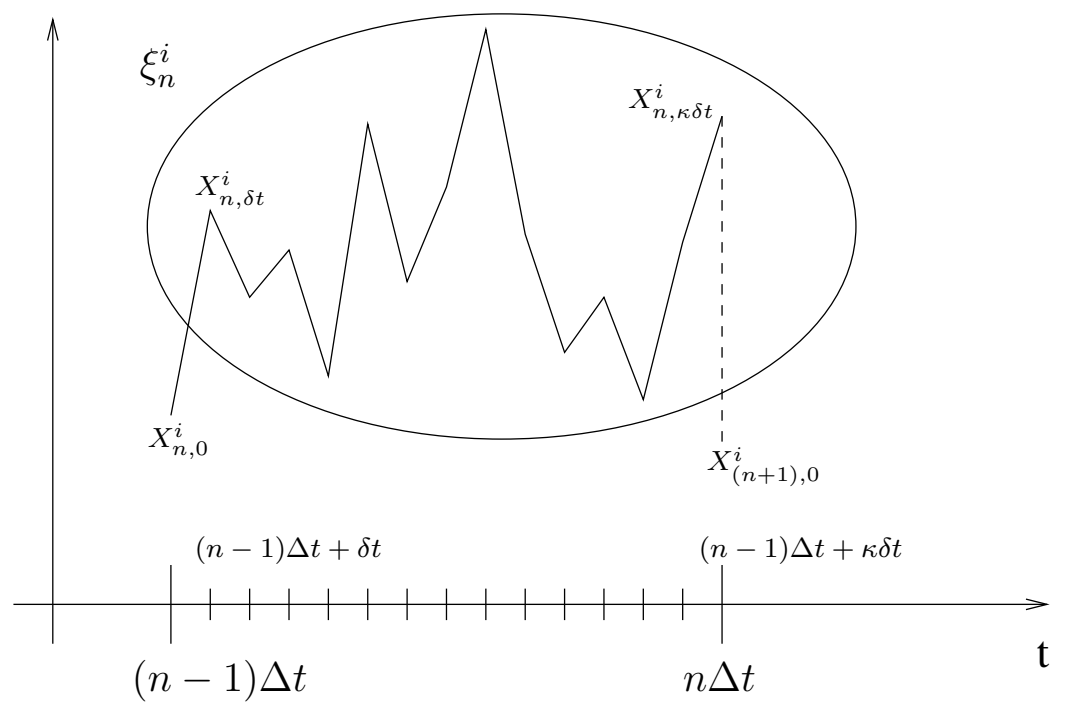

Figure 1. The $i$-th particle $\xi_{n}^{i}$ at time index $n$ is composed of the successive positions $\left(X_{n, \delta t}^{i}, \ldots, X_{n, \kappa \delta t}^{i}\right)$ of the $i$-th walker on time interval $((n-1) \Delta t, n \Delta t)$.

Here $\left(W^{1}, \ldots, W^{N}\right)$ denotes a $N$-dimensional Brownian motion independent from the initial positions of the walkers $\left(X_{1,0}^{i}\right)_{1 \leq i \leq N}$ which are i.i.d. according to $2 \psi_{I}^{2}(x) 1_{\{x>0\}} \mathrm{d} x$. We recall that in our framework, it is possible to simulate exactly in law all these random variables (see Appendix). We store the successive positions $\left(X_{n, \delta t}^{i}, \ldots, X_{n, \kappa \delta t}^{i}\right)$ of the $i$-th walker over the time interval $((n-1) \Delta t, n \Delta t)$ in a so-called particle $\xi_{n}^{i} \in\left(\mathbb{R}_{+}^{*}\right)^{\kappa}$ (see Fig. 1): $\forall i \in\{1, \ldots, N\}, \forall n \in\{1, \ldots, \nu\}$,

$$
\xi_{n}^{i}=\left(X_{n, \delta t}^{i}, \ldots, X_{n, \kappa \delta t}^{i}\right) .
$$

In the following, we will denote by $\xi_{n}=\left(\xi_{n}^{1}, \ldots, \xi_{n}^{N}\right)$ the configuration of the ensemble of particles at time index $n$. We have here described the mutation step.

For a given configuration of the particles $\xi_{n}$ at a time index $n \in\{1, \ldots, \nu\}$, the selection step now consists in choosing the initial positions $\left(X_{n+1,0}^{i}\right)_{1 \leq i \leq N}$ of the $N$ walkers at time $n \Delta t$ using one of the following resampling algorithm:

(S1) The $\left(X_{n+1,0}^{i}\right)_{1 \leq i \leq N}$ are conditionally independent w.r.t. $\xi_{n}$ and for $1 \leq i \leq N, X_{n+1,0}^{i}$ is distributed according to the measure

$$
\epsilon_{n} g\left(\xi_{n}^{i}\right) \delta_{\xi_{n, \kappa}^{i}}+\left(1-\epsilon_{n} g\left(\xi_{n}^{i}\right)\right) \sum_{j=1}^{N} \rho_{n}^{j} \delta_{\xi_{n, \kappa}^{j}}
$$

where $g$ is defined by, for $y=\left(y_{1}, \ldots, y_{\kappa}\right) \in\left(\mathbb{R}_{+}^{*}\right)^{\kappa}$,

$$
g(y)=\exp \left(-\theta \delta t \sum_{k=1}^{\kappa} y_{k}^{4}\right),
$$

$\rho_{n}^{j}$ denotes the weight of the $j$-th particle

$$
\rho_{n}^{j}=\frac{g\left(\xi_{n}^{j}\right)}{\sum_{j=1}^{N} g\left(\xi_{n}^{j}\right)}
$$


and $\epsilon_{n}$ is a non negative function of $\xi_{n}$ such that $\epsilon_{n} \leq 1 / \max _{1 \leq i \leq N} g\left(\xi_{n}^{i}\right)$. In particular the following choices are possible for $\epsilon_{n}$ :

$$
\epsilon_{n}=0, \epsilon_{n}=1 \text { or } \epsilon_{n}=\frac{1}{\max _{1 \leq i \leq N} g\left(\xi_{n}^{i}\right)} .
$$

The so-called multinomial resampling method which corresponds to the choice $\epsilon_{n}=0$ gives rise to a maximum decorrelation with the former position of the particles, while with growing $\epsilon_{n}$, more and more correlation is introduced.

(S2) The $\left(X_{n+1,0}^{i}\right)_{1 \leq i \leq N}$ are such that

$$
\left\{\begin{array}{l}
\forall j \in\{1, \ldots, N\}, \forall i \in\left\{\left(1+\sum_{l=1}^{j-1} a_{n}^{l}\right), \ldots,\left(\sum_{l=1}^{j} a_{n}^{l}\right)\right\}, \\
\quad X_{n+1,0}^{i}=\xi_{n, \kappa}^{j}, \\
\text { and the variables }\left(X_{n+1,0}^{i}\right)_{1+\sum_{l=1}^{N} a_{n}^{l} \leq i \leq N} \text { are conditionally independent w.r.t. } \xi_{n}, \\
\quad \text { with } X_{n+1,0}^{i} \text { distributed according to } \sum_{j=1}^{N}\left\{N \rho_{n}^{j}\right\} \delta_{\xi_{n, \kappa}^{j}} /\left(N-\sum_{l=1}^{N} a_{n}^{l}\right),
\end{array}\right.
$$

where

$$
a_{n}^{j}=\left\lfloor N \rho_{n}^{j}\right\rfloor, j \in\{1, \ldots, N\} .
$$

Notice that the $\left(X_{n+1,0}^{i}\right)_{1 \leq i \leq N}$ are conditionally independent w.r.t. $\xi_{n}$. This is the so-called residual resampling method.

(S3) The $\left(X_{n+1,0}^{i}\right)_{1 \leq i \leq N}$ are such that, for $1 \leq i \leq N$,

$$
X_{(n+1), 0}^{i}=\sum_{j=1}^{N} 1_{\left\{\sum_{l=1}^{j-1} \rho_{n}^{l}<\left(i-U_{n}^{i}\right) / N \leq \sum_{l=1}^{j} \rho_{n}^{l}\right\}} \xi_{n, \kappa}^{j},
$$

where $\left(U_{n}^{i}\right)_{1 \leq i \leq N}$ are random variables i.i.d. according to the uniform law on $[0,1]$, independently of $\xi_{n}$. Notice that the $\left(X_{n+1,0}^{i}\right)_{1 \leq i \leq N}$ are conditionally independent w.r.t. $\xi_{n}$. This is the so-called stratified resampling method.

For $n \in\{1, \ldots, \nu\}$, let us denote by

$$
\eta_{n}^{N}=\frac{1}{N} \sum_{i=1}^{N} \delta_{\xi_{n}^{i}}
$$

the particle approximation of the measure $\eta_{n}$ defined by: $\forall f:\left(\mathbb{R}_{+}^{*}\right)^{\kappa} \rightarrow \mathbb{R}$ bounded,

$$
\eta_{n}(f)=\frac{\mathbb{E}\left(f\left(X_{(n-1) \Delta t+\delta t}, \ldots, X_{(n-1) \Delta t+\kappa \delta t}\right) \exp \left(-\theta \delta t \sum_{k=1}^{(n-1) \kappa}\left(X_{k \delta t}\right)^{4}\right)\right)}{\mathbb{E}\left(\exp \left(-\theta \delta t \sum_{k=1}^{(n-1) \kappa}\left(X_{k \delta t}\right)^{4}\right)\right)}
$$

where the process $\left(X_{t}\right)_{0 \leq t \leq T}$ is defined by (15).

For $y=\left(y_{1}, \ldots, y_{\kappa}\right) \in\left(\overline{\mathbb{R}}_{+}^{*}\right)^{\kappa}$ and $f:\left(\mathbb{R}_{+}^{*}\right)^{\kappa} \rightarrow \mathbb{R}$, we set

$$
P f(y)=\mathbb{E}\left(f\left(X_{\delta t}^{y_{\kappa}}, \ldots, X_{\kappa \delta t}^{y_{\kappa}}\right)\right)
$$

where for $x \in \mathbb{R}_{+}^{*}$,

$$
X_{t}^{x}=x+\int_{0}^{t} b\left(X_{s}^{x}\right) \mathrm{d} s+W_{t}
$$


denotes the solution of the stochastic differential equation (15) starting from $x$. By the Markov property, the measures $\left(\eta_{n}\right)_{1 \leq n \leq \nu}$ satisfy the inductive relations, for any function $f:\left(\mathbb{R}_{+}^{*}\right)^{\kappa} \rightarrow \mathbb{R}$ bounded, $\forall n \in\{1, \ldots, \nu-1\}$,

$$
\begin{aligned}
\eta_{n+1}(f) & =\frac{\mathbb{E}\left(\exp \left(-\theta \delta t \sum_{k=1}^{n \kappa}\left(X_{k \delta t}\right)^{4}\right) \mathbb{E}\left(f\left(X_{n \Delta t+\delta t}, \ldots, X_{n \Delta t+\kappa \delta t}\right)\left(X_{j \delta t}\right)_{0 \leq j \leq n \kappa}\right)\right)}{\eta_{n}(g) \mathbb{E}\left(\exp \left(-\theta \delta t \sum_{k=1}^{(n-1) \kappa}\left(X_{k \delta t}\right)^{4}\right)\right)} \\
& =\frac{1}{\eta_{n}(g)} \times \frac{\mathbb{E}\left(g P f\left(X_{(n-1) \Delta t+\delta t}, \ldots, X_{(n-1) \Delta t+\kappa \delta t}\right) \exp \left(-\theta \delta t \sum_{k=1}^{(n-1) \kappa}\left(X_{k \delta t}\right)^{4}\right)\right)}{\mathbb{E}\left(\exp \left(-\theta \delta t \sum_{k=1}^{(n-1) \kappa}\left(X_{k \delta t}\right)^{4}\right)\right)}=\frac{\eta_{n}(g P f)}{\eta_{n}(g)},
\end{aligned}
$$

where $g$ is defined by $(24)$. Moreover, we can express $E_{\mathrm{DMC}}^{\delta t}(T)$ defined by (16) as:

$$
E_{\mathrm{DMC}}^{\delta t}(T)=\frac{3}{2} \omega+\theta \frac{\eta_{\nu}\left(g y_{\kappa}^{4}\right)}{\eta_{\nu}(g)}
$$

Therefore the particle approximation of $E_{\mathrm{DMC}}(T)$ is given by

$$
E_{\mathrm{DMC}}^{N, \nu, \kappa}(T)=\frac{3}{2} \omega+\theta \frac{\eta_{\nu}^{N}\left(g y_{\kappa}^{4}\right)}{\eta_{\nu}^{N}(g)}
$$

This approximation of $E_{\mathrm{DMC}}(T)$ corresponds to the expression (8) given in the introduction. We will also prove in Corollary 13 below the convergence of the approximation which corresponds to the expression (7) given in the introduction (see Eq. (46) below).

The convergence of the approximation $E_{\mathrm{DMC}}^{N, \nu, \kappa}(T)$ is ensured by our main result:

\section{Theorem 4.}

$$
\mathbb{E}\left|E_{\mathrm{DMC}}(T)-E_{\mathrm{DMC}}^{N, \nu, \kappa}(T)\right| \leq \frac{C}{\nu \kappa}+\frac{C_{\nu}}{\sqrt{N}},
$$

where the constant $C$ only depends on $T$ and the constant $C_{\nu}$ on $T$ and $\nu$.

Remark 5. The number of selection steps is $\nu-1$. For instance, when $\nu=1$, there is no selection involved in the expression of $E_{\mathrm{DMC}}^{N, \nu, \kappa}(T)$ and the particles remain independent. In this case, the first term in the right hand side of (38) corresponds to the time discretization error proved in Proposition 1, while the second term is the classical error estimate related to the law of large numbers. For a fixed number of selection steps, the theorem ensures the convergence of the particle approximation $E_{\mathrm{DMC}}^{N, \nu, \kappa}(T)$ as the time-step $\delta t=T /(\nu \kappa)$ used for the discretization of the stochastic differential equation (15) tends to 0 while the number $N$ of particles tends to $+\infty$. But this result does not specify the dependence of $C_{\nu}$ on $\nu$ and gives no hint on the optimal choice of the number of selection steps in terms of error minimization. We are going to deal with this important issue in the numerical study (see Sect. 2).

According to the above expressions (36) and (37) of $E_{\mathrm{DMC}}^{\delta t}(T)$ and $E_{\mathrm{DMC}}^{N, \nu, \kappa}(T)$, this theorem is easily proved by combining Proposition 1 and the following result:

\section{Proposition 6.}

$$
\mathbb{E}\left|\frac{\eta_{\nu}^{N}\left(g y_{\kappa}^{4}\right)}{\eta_{\nu}^{N}(g)}-\frac{\eta_{\nu}\left(g y_{\kappa}^{4}\right)}{\eta_{\nu}(g)}\right| \leq \frac{C_{\nu}}{\sqrt{N}}
$$


Proof. One has

$$
\begin{aligned}
\mathbb{E}\left|\frac{\eta_{\nu}^{N}\left(g y_{\kappa}^{4}\right)}{\eta_{\nu}^{N}(g)}-\frac{\eta_{\nu}\left(g y_{\kappa}^{4}\right)}{\eta_{\nu}(g)}\right| \leq & \frac{\mathbb{E}\left|\eta_{\nu}^{N}\left(g y_{\kappa}^{4}\right)-\eta_{\nu}\left(g y_{\kappa}^{4}\right)\right|}{\eta_{\nu}(g)} \\
& +\left(\mathbb{E}\left(\frac{\eta_{\nu}^{N}\left(g y_{\kappa}^{4}\right)}{\eta_{\nu}^{N}(g)}\right)^{2}\right)^{1 / 2} \frac{\left(\mathbb{E}\left(\eta_{\nu}^{N}(g)-\eta_{\nu}(g)\right)^{2}\right)^{1 / 2}}{\eta_{\nu}(g)}
\end{aligned}
$$

According to Proposition 7 and Lemma 12 below, the first term of the right-hand-side and the quotient in the second term are smaller than $C_{\nu} / \sqrt{N}$. Since by Jensen's inequality, $\left(\frac{\eta_{\nu}^{N}\left(g y_{\kappa}^{4}\right)}{\eta_{\nu}^{N}(g)}\right)^{2} \leq \frac{\eta_{\nu}^{N}\left(g y_{\kappa}^{8}\right)}{\eta_{\nu}^{N}(g)}$, the boundedness of $\mathbb{E}\left(\frac{\eta_{\nu}^{N}\left(g y_{\kappa}^{4}\right)}{\eta_{\nu}^{N}(g)}\right)^{2}$ follows from Lemma 9 below.

Proposition 7. For any bounded function $f:\left(\mathbb{R}_{+}^{*}\right)^{\kappa} \rightarrow \mathbb{R}$,

$$
\forall n \in\{1, \ldots, \nu\}, \mathbb{E}\left(\left(\eta_{n}^{N}(f)-\eta_{n}(f)\right)^{2}\right) \leq \frac{C_{n}}{N}\|f\|_{\infty}^{2}
$$

where the constant $C_{n}$ does not depend on $\kappa$.

For any function $f:\left(\mathbb{R}_{+}^{*}\right)^{\kappa} \rightarrow \mathbb{R}$ such that for some $p \geq 2,\|f\|_{\kappa, p}=\sup _{y \in \mathbb{R}_{+}^{\kappa}} \frac{|f(y)|}{1+y_{\kappa}^{p}}$ is finite,

$$
\forall n \in\{1, \ldots, \nu\}, \mathbb{E}\left|\eta_{n}^{N}(f)-\eta_{n}(f)\right| \leq \frac{C_{n}}{\sqrt{N}}\|f\|_{\kappa, p},
$$

where the constant $C_{n}$ does not depend on $\kappa$.

For $f$ bounded, the first estimate (40) is proved in [9]. In order to prove Proposition 6, we need to apply Proposition 7 with $f(y)=g(y)$ and $f(y)=g(y) y_{\kappa}^{4}$, which are bounded functions with $L^{\infty}$ norm respectively equal to 1 and $\frac{C}{\delta t}$ where $C$ is a constant not depending on $\delta t$. But we want to obtain the convergence when $\delta t$ tends to 0 . This is why we need the second estimate $(41)$, that we use with $f(y)=g(y) y_{\kappa}^{4}$ for which $\|f\|_{\kappa, p}$ is bounded and does not depend on $\delta t$.

Notice that for $f$ bounded, Corollary 2.20 in [9] states the convergence in law of $\sqrt{N}\left(\eta_{n}^{N}(f)-\eta_{n}(f)\right)$ to a centered Gaussian variable and gives an expression of the variance of this limit variable. Because of the complexity of this expression, using this result with $f(y)=g(y) y_{\kappa}^{4}$ did not really help us to understand the dependence of $C_{\nu}$ on $\nu$ (see Rem. 5 above).

Proof. For $f$ bounded, the first estimate (40) is proved by induction on $n$ in [9] (see Prop. 2.9). Since we follow the same inductive reasoning to deal with $f$ such that $\|f\|_{\kappa, p}<+\infty$, we give at the same time the proof for $f$ bounded.

Since the initial positions $\left(\xi_{1}^{i}\right)_{1 \leq i \leq N}$ are independent and identically distributed with $\xi_{1, \kappa}^{i}$ distributed according to $2 \psi_{I}^{2}(x) 1_{\{x>0\}} \mathrm{d} x$, the statement holds for $n=1$.

To deduce the statement at rank $n+1$ from the statement at rank $n$, we remark that according to (35),

$$
\eta_{n+1}^{N}(f)-\eta_{n+1}(f)=T_{n+1}+\frac{1}{\eta_{n}(g)}\left(\left(\eta_{n}^{N}(g P f)-\eta_{n}(g P f)\right)+\frac{\eta_{n}^{N}(g P f)}{\eta_{n}^{N}(g)}\left(\eta_{n}(g)-\eta_{n}^{N}(g)\right)\right)
$$

where we recall that $P$ is defined by $(32)$, and

$$
T_{n+1}=\eta_{n+1}^{N}(f)-\frac{\eta_{n}^{N}(g P f)}{\eta_{n}^{N}(g)}
$$


To deal with this term $T_{n+1}$, one remarks that for the first type of selection step (S1), all the possible choices of $\epsilon_{n}$ given in $(26)$ are $\sigma\left(\xi_{n}\right)$-measurable. As a consequence, for $i \in\{1, \ldots, N\}$,

$$
\mathbb{E}\left(f\left(\xi_{n+1}^{i}\right) \mid \xi_{n}\right)=\epsilon_{n} g\left(\xi_{n}^{i}\right) \operatorname{Pf}\left(\xi_{n}^{i}\right)+\left(1-\epsilon_{n} g\left(\xi_{n}^{i}\right)\right) \sum_{j=1}^{N} \rho_{n}^{j} P f\left(\xi_{n}^{j}\right)
$$

where $\rho_{n}^{j}$ is defined by (25). Multiplying this equality by $\frac{1}{N}$ and summing over $i$, one deduces

$$
\mathbb{E}\left(\eta_{n+1}^{N}(f) \mid \xi_{n}\right)=\sum_{j=1}^{N} \rho_{n}^{j} \operatorname{Pf}\left(\xi_{n}^{j}\right)=\frac{\sum_{j=1}^{N} g\left(\xi_{n}^{j}\right) P f\left(\xi_{n}^{j}\right)}{\sum_{j=1}^{N} g\left(\xi_{n}^{j}\right)}=\frac{\eta_{n}^{N}(g P f)}{\eta_{n}^{N}(g)} .
$$

Now, for the stochastic remainder resampling algorithm (S2), by $(27), \mathbb{E}\left(\eta_{n+1}^{N}(f) \mid \xi_{n}\right)$ is equal to

$$
\frac{1}{N} \sum_{j=1}^{N}\left\lfloor\frac{N g\left(\xi_{n}^{j}\right)}{\sum_{l=1}^{N} g\left(\xi_{n}^{l}\right)}\right\rfloor \operatorname{Pf}\left(\xi_{n}^{j}\right)+\sum_{i=1+\sum_{l=1}^{N} a_{n}^{l}}^{N} \frac{1}{N-\sum_{l=1}^{N} a_{n}^{l}} \sum_{j=1}^{N}\left\{\frac{N g\left(\xi_{n}^{j}\right)}{\sum_{l=1}^{N} g\left(\xi_{n}^{l}\right)}\right\} \operatorname{Pf}\left(\xi_{n}^{j}\right)
$$

and (43) still holds. Finally, for the stratified resampling method (S3), by (29), we have (using the footnote ${ }^{2}$ )

$$
\begin{aligned}
\mathbb{E}\left(\eta_{n+1}^{N}(f) \mid \xi_{n}\right) & =\frac{1}{N} \sum_{i=1}^{N} \sum_{j=1}^{N} \mathbb{E}\left(1_{\left\{\sum_{l=1}^{j-1} \rho_{n}^{l}<\left(i-U_{n}^{i}\right) / N \leq \sum_{l=1}^{j} \rho_{n}^{l}\right\}} \mid \xi_{n}\right) \operatorname{Pf}\left(\xi_{n}^{j}\right), \\
& =\frac{1}{N} \sum_{j=1}^{N} \mathbb{E}\left(\sum_{i=1}^{N} 1_{\left\{\sum_{l=1}^{j-1} \rho_{n}^{l}<\left(i-U_{n}^{1}\right) / N \leq \sum_{l=1}^{j} \rho_{n}^{l}\right\}} \mid \xi_{n}\right) \operatorname{Pf}\left(\xi_{n}^{j}\right), \\
& =\frac{1}{N} \sum_{j=1}^{N} \mathbb{E}\left(\left\lfloor N \sum_{l=1}^{j} \rho_{n}^{l}+U_{n}^{1}\right\rfloor-\left\lfloor N \sum_{l=1}^{j-1} \rho_{n}^{l}+U_{n}^{1}\right\rfloor \mid \xi_{n}\right) \operatorname{Pf}\left(\xi_{n}^{j}\right), \\
& =\sum_{j=1}^{N} \rho_{n}^{j} \operatorname{Pf}\left(\xi_{n}^{j}\right),
\end{aligned}
$$

which yields again (43). Since for all three possible selection steps, the variables $\left(\xi_{n+1}^{i}\right)_{1 \leq i \leq N}$ are independent conditionally on $\xi_{n}$, one deduces that

$$
\mathbb{E}\left(\left(T_{n+1}\right)^{2} \mid \xi_{n}\right)=\frac{1}{N^{2}} \sum_{i=1}^{N} \mathbb{E}\left(\left(f\left(\xi_{n+1}^{i}\right)-\mathbb{E}\left(f\left(\xi_{n+1}^{i}\right) \mid \xi_{n}\right)\right)^{2} \mid \xi_{n}\right) \leq \frac{1}{N} \mathbb{E}\left(\eta_{n+1}^{N}\left(f^{2}\right) \mid \xi_{n}\right)
$$

Therefore

$$
\mathbb{E}\left(\left(T_{n+1}\right)^{2}\right) \leq \frac{1}{N} \mathbb{E}\left(\eta_{n+1}^{N}\left(f^{2}\right)\right) .
$$

When $f$ is bounded, $\eta_{n+1}^{N}\left(f^{2}\right) \leq\|f\|_{\infty}^{2},\left|\frac{\eta_{n}^{N}(g P f)}{\eta_{n}^{N}(g)}\right| \leq\|P f\|_{\infty}$, and $\|P f\|_{\infty} \leq\|f\|_{\infty}$. Hence by (42),

$$
\mathbb{E}\left(\left(\eta_{n+1}^{N}(f)-\eta_{n+1}(f)\right)^{2}\right) \leq 3\left(\frac{\|f\|_{\infty}^{2}}{N}+\frac{\mathbb{E}\left(\left(\eta_{n}^{N}(g P f)-\eta_{n}(g P f)\right)^{2}\right)+\|f\|_{\infty}^{2} \mathbb{E}\left(\left(\eta_{n}^{N}(g)-\eta_{n}(g)\right)^{2}\right)}{\left(\eta_{n}(g)\right)^{2}}\right)
$$

with the second term of the right-hand-side smaller than $C\|f\|_{\infty}^{2} / N$ by the induction hypothesis and Lemma 12 below. 
When $\|f\|_{\kappa, p}<+\infty$, combining (42) and (44), one obtains

$$
\begin{aligned}
\mathbb{E}\left|\eta_{n+1}^{N}(f)-\eta_{n+1}(f)\right| \leq & \frac{\left(\mathbb{E}\left(\eta_{n+1}^{N}\left(f^{2}\right)\right)\right)^{1 / 2}}{\sqrt{N}}+\frac{\mathbb{E}\left|\eta_{n}^{N}(g P f)-\eta_{n}(g P f)\right|}{\eta_{n}(g)} \\
& +\left(\mathbb{E}\left(\frac{\eta_{n}^{N}(g P f)}{\eta_{n}^{N}(g)}\right)^{2}\right)^{1 / 2} \frac{\left(\mathbb{E}\left(\eta_{n}^{N}(g)-\eta_{n}(g)\right)^{2}\right)^{1 / 2}}{\eta_{n}(g)}
\end{aligned}
$$

Since $\left\|f^{2}\right\|_{k, 2 p} \leq 2\|f\|_{k, p}^{2}$ (by using the inequality $f^{2}(y) \leq 2\|f\|_{\kappa, p}^{2}\left(1+y_{\kappa}^{2 p}\right)$ ), the first term of the right-handside is smaller than $C_{n}\|f\|_{\kappa, p} / \sqrt{N}$ by Lemma 9 below. Since, according to Lemma 10 below, $\|P f\|_{\kappa, p} \leq$ $\mathrm{e}^{C_{p} \Delta t}\|f\|_{\kappa, p}$, the second term is smaller than $C_{n}\|f\|_{\kappa, p} / \sqrt{N}$ by the induction hypothesis and Lemma 12. Last, by using successively Cauchy Schwartz inequalities, (43) for $f^{2}$ and Lemma 9, one obtains that $\mathbb{E}\left(\frac{\eta_{n}^{N}(g P f)}{\eta_{n}^{N}(g)}\right)^{2} \leq$ $\mathbb{E}\left(\frac{\eta_{n}^{N}\left(g(P f)^{2}\right)}{\eta_{n}^{N}(g)}\right) \leq \mathbb{E}\left(\frac{\eta_{n}^{N}\left(g P f^{2}\right)}{\eta_{n}^{N}(g)}\right)=\mathbb{E}\left(\eta_{n+1}^{N}\left(f^{2}\right)\right) \leq C_{n}\|f\|_{\kappa, p}^{2}$. And it follows from the proposition statement for $f$ bounded and Lemma 12 that $\frac{\left(\mathbb{E}\left(\eta_{n}^{N}(g)-\eta_{n}(g)\right)^{2}\right)^{1 / 2}}{\eta_{n}(g)}$ is smaller than $C_{n} / \sqrt{N}$.

Remark 8. Proposition 7 (and therefore Thm. 4) also hold for the stratified remainder resampling algorithm, which consists in combining the stochastic remainder resampling and the stratified resampling. More precisely, it consists in replicating $\left\lfloor N \rho_{n}^{i}\right\rfloor$ times the $i$-th particle, and then completing the set of particles by using stratified resampling to draw the $N^{R}=N-\sum_{l=1}^{N}\left\lfloor N \rho_{n}^{l}\right\rfloor$ remaining particles, the $i$-th particle being assigned the weight $\rho_{n}^{R, i}=\left\{N \rho_{n}^{i}\right\} / N^{R}$.

Lemma 9. Let $h:\left(\mathbb{R}_{+}^{*}\right)^{\kappa} \rightarrow \mathbb{R}_{+}$be such that for some $p \geq 2$, $\|h\|_{\kappa, p}<+\infty$. Then,

$$
\forall n \in\{1, \ldots, \nu\}, \max \left(\mathbb{E}\left(\eta_{n}^{N}(h)\right), \mathbb{E}\left(\frac{\eta_{n}^{N}(g h)}{\eta_{n}^{N}(g)}\right)\right) \leq \mathrm{e}^{C_{p} n \Delta t}\|h\|_{\kappa, p}\left(1+\mathbb{E}\left(X_{0}\right)^{p}\right),
$$

where $X_{0}$ is distributed according to the measure $2 \psi_{I}^{2}(x) 1_{\{x>0\}} \mathrm{d} x$ (see (15)).

Proof. As the variables $\xi_{1, \kappa}^{i}, 1 \leq i \leq N$ are distributed according to the invariant measure $2 \psi_{I}^{2}(x) 1_{\{x>0\}} \mathrm{d} x$, one has $\mathbb{E}\left(\eta_{1}^{N}(h)\right) \leq\|h\|_{\kappa, p}\left(1+\mathbb{E}\left(X_{0}\right)^{p}\right)$. In addition for $n \geq 1$, according to $(43), \mathbb{E}\left(\eta_{n+1}^{N}(h)\right)=\mathbb{E}\left(\frac{\eta_{n}^{N}(g P h)}{\eta_{n}^{N}(g)}\right)$ where $\|P h\|_{\kappa, p} \leq \mathrm{e}^{C_{p} \Delta t}\|h\|_{k, p}$ by Lemma 10. Therefore it is enough to check the bound for $\mathbb{E}\left(\frac{\eta_{n}^{N}(g h)}{\eta_{n}^{N}(g)}\right)$.

For $n \geq 0$, one has

$$
\mathbb{E}\left(\frac{\eta_{n+1}^{N}(g h)}{\eta_{n+1}^{N}(g)}\right) \leq\|h\|_{\kappa, p}\left(1+\mathbb{E}\left(\frac{\sum_{i=1}^{N} \exp \left(-\theta \delta t \sum_{k=1}^{\kappa}\left(\xi_{n+1, k}^{i}\right)^{4}\right)\left(\xi_{n+1, \kappa}^{i}\right)^{p}}{\sum_{j=1}^{N} \exp \left(-\theta \delta t \sum_{k=1}^{\kappa}\left(\xi_{n+1, k}^{j}\right)^{4}\right)}\right)\right) .
$$


Let us denote in this proof $\xi_{n+1,0}^{i}=X_{n+1,0}^{i}$, where $0 \leq n \leq \nu-1$ and $1 \leq i \leq N$. Let us set $\mathcal{F}=\sigma\left(\xi_{n+1, k}^{i}\right.$, $1 \leq i \leq N, 0 \leq k \leq \kappa-1)$. By Lemma 11 below,

$$
\begin{gathered}
\mathbb{E}\left(\frac{\sum_{i=1}^{N} \exp \left(-\theta \delta t \sum_{k=1}^{\kappa}\left(\xi_{n+1, k}^{i}\right)^{4}\right)\left(\xi_{n+1, \kappa}^{i}\right)^{p}}{\sum_{j=1}^{N} \exp \left(-\theta \delta t \sum_{k=1}^{\kappa}\left(\xi_{n+1, k}^{j}\right)^{4}\right)} \mid \mathcal{F}\right) \leq \frac{\sum_{i=1}^{N} \exp \left(-\theta \delta t \sum_{k=1}^{\kappa-1}\left(\xi_{n+1, k}^{i}\right)^{4}\right) \mathbb{E}\left(\left(\xi_{n+1, \kappa}^{i}\right)^{p} \mid \mathcal{F}\right)}{\sum_{j=1}^{N} \exp \left(-\theta \delta t \sum_{k=1}^{\kappa-1}\left(\xi_{n+1, k}^{j}\right)^{4}\right)}, \\
=\frac{\left.\sum_{i=1}^{N} \exp \left(-\theta \delta t \sum_{k=1}^{\kappa-1}\left(\xi_{n+1, k}^{i}\right)^{4}\right) \mathbb{E}\left(\left(X_{\delta t}^{x}\right)^{p}\right)\right|_{x=\xi_{n+1, \kappa-1}^{i}}}{\sum_{j=1}^{N} \exp \left(-\theta \delta t \sum_{k=1}^{\kappa-1}\left(\xi_{n+1, k}^{j}\right)^{4}\right)} \\
\leq \mathrm{e}^{C_{p} \delta t} \frac{\sum_{i=1}^{N} \exp \left(-\theta \delta t \sum_{k=1}^{\kappa-1}\left(\xi_{n+1, k}^{i}\right)^{4}\right)\left(\xi_{n+1, \kappa-1}^{i}\right)^{p}}{\sum_{j=1}^{N} \exp \left(-\theta \delta t \sum_{k=1}^{\kappa-1}\left(\xi_{n+1, k}^{j}\right)^{4}\right)}+\mathrm{e}^{C_{p} \delta t}-1,
\end{gathered}
$$

where we have used the definition of the mutation step (see (21)) and the Markov property for the stochastic differential equation (33) to obtain the equality, and then Lemma 10 for the last inequality. Notice that this estimate also holds for $\kappa=1$, in which case the right hand side reduces to $\frac{\mathrm{e}^{C_{p} \delta t}}{N}\left(\xi_{n+1,0}^{i}\right)^{p}+\mathrm{e}^{C_{p} \delta t}-1$.

Taking expectations and iterating the reasoning, one deduces that

$$
\mathbb{E}\left(\frac{\sum_{i=1}^{N} \exp \left(-\theta \delta t \sum_{k=1}^{\kappa}\left(\xi_{n+1, k}^{i}\right)^{4}\right)\left(\xi_{n+1, \kappa}^{i}\right)^{p}}{\sum_{j=1}^{N} \exp \left(-\theta \delta t \sum_{k=1}^{\kappa}\left(\xi_{n+1, k}^{j}\right)^{4}\right)}\right) \leq \frac{\mathrm{e}^{C_{p} \Delta t}}{N} \sum_{i=1}^{N} \mathbb{E}\left(\left(\xi_{n+1,0}^{i}\right)^{p}\right)+\left(\mathrm{e}^{C_{p} \delta t}-1\right) \sum_{k=0}^{\kappa-1} \mathrm{e}^{C_{p} k \delta t} .
$$

Inserting this bound in (45), one concludes that

$$
\mathbb{E}\left(\frac{\eta_{n+1}^{N}(g h)}{\eta_{n+1}^{N}(g)}\right) \leq \mathrm{e}^{C_{p} \Delta t}\|h\|_{\kappa, p}\left(1+\mathbb{E}\left(\frac{1}{N} \sum_{i=1}^{N}\left(\xi_{n+1,0}^{i}\right)^{p}\right)\right) .
$$

For $n=0$, one deduces that $\mathbb{E}\left(\frac{\eta_{1}^{N}(g h)}{\eta_{1}^{N}(g)}\right) \leq \mathrm{e}^{C_{p} \Delta t}\|h\|_{\kappa, p}\left(1+\mathbb{E}\left(X_{0}^{p}\right)\right)$, where $X_{0}$ is distributed according to the measure $2 \psi_{I}^{2}(x) 1_{\{x>0\}} \mathrm{d} x$.

For $n \geq 1$, since by a reasoning similar to the one made to obtain (43), $\mathbb{E}\left(\frac{1}{N} \sum_{i=1}^{N}\left(\xi_{n+1,0}^{i}\right)^{p}\right)=\mathbb{E}\left(\frac{\eta_{n}^{N}\left(g(y) y_{\kappa}^{p}\right)}{\eta_{n}^{N}(g(y))}\right)$, one also deduces that

$$
\mathbb{E}\left(\frac{\eta_{n+1}^{N}(g h)}{\eta_{n+1}^{N}(g)}\right) \leq \mathrm{e}^{C_{p} \Delta t}\|h\|_{\kappa, p} \mathbb{E}\left(\frac{\eta_{n}^{N}\left(g\left(1+y_{\kappa}^{p}\right)\right)}{\eta_{n}^{N}(g)}\right) .
$$

The proof is completed by an obvious inductive reasoning.

Lemma 10. For any $p \geq 2$, there is a constant $C_{p}$ such that

$$
\forall x \in \mathbb{R}_{+}^{*}, \forall t \geq 0, \mathbb{E}\left(\left(X_{t}^{x}\right)^{p}\right) \leq\left(1+x^{p}\right) \mathrm{e}^{C_{p} t}-1,
$$

where $X_{t}^{x}$ is defined by (33). Therefore, if $h:\left(\mathbb{R}_{+}^{*}\right)^{\kappa} \rightarrow \mathbb{R}$ is such that $\|h\|_{\kappa, p}<+\infty$ then $\|P h\|_{\kappa, p} \leq \mathrm{e}^{C_{p} \Delta t}\|h\|_{\kappa, p}$, where the operator $P$ is defined by (32). 
Proof. By Itô's formula, $\mathrm{d}\left(X_{t}^{x}\right)^{p}=\left(\frac{p(p+1)}{2}\left(X_{t}^{x}\right)^{p-2}-\omega p\left(X_{t}^{x}\right)^{p}\right) \mathrm{d} t+p\left(X_{t}^{x}\right)^{p-1} \mathrm{~d} W_{t}$. Hence

$$
\left(X_{t}^{x}\right)^{p} \leq x^{p}+\int_{0}^{t}\left(\frac{p(p+1)}{2}+\frac{p(p+1-2 \omega)}{2}\left(X_{s}^{x}\right)^{p}\right) \mathrm{d} s+p \int_{0}^{t}\left(X_{s}^{x}\right)^{p-1} \mathrm{~d} W_{s} .
$$

Formally, taking expectations in this inequality, one obtains

$$
\mathbb{E}\left(\left(X_{t}^{x}\right)^{p}\right) \leq x^{p}+\int_{0}^{t} \frac{p(p+1)}{2}+\frac{p(p+1-2 \omega)}{2} \mathbb{E}\left(\left(X_{s}^{x}\right)^{p}\right) \mathrm{d} s
$$

and check by Gronwall's lemma that the conclusion holds with $C_{p}=\frac{p(p+1)}{2}$. This formal argument can be made rigorous by a standard localization procedure.

For $h: \mathbb{R}_{+}^{\kappa} \rightarrow \mathbb{R}$ such that $\|h\|_{\kappa, p}<+\infty$ one deduces that

$$
\forall y \in \mathbb{R}_{+}^{\kappa},|P h(y)| \leq \mathbb{E}\left|h\left(X_{\delta t}^{y_{\kappa}}, \ldots, X_{\kappa \delta t}^{y_{\kappa}}\right)\right| \leq C\|h\|_{\kappa, p}\left(1+\mathbb{E}\left(\left(X_{\kappa \delta t}^{y_{\kappa}}\right)^{p}\right)\right) \leq \mathrm{e}^{C_{p} \Delta t}\|h\|_{\kappa, p}\left(1+y_{\kappa}^{p}\right)
$$

\section{Lemma 11.}

$$
\forall\left(z_{1}, \ldots, z_{N}\right),\left(a_{1}, \ldots, a_{N}\right) \in \mathbb{R}_{+}^{N} \text { with } \sum_{i=1}^{N} a_{i}>0, \forall p \geq 0, \forall c \geq 0, \frac{\sum_{i=1}^{N} a_{i} z_{i}^{p} \mathrm{e}^{-c z_{i}^{4}}}{\sum_{i=1}^{N} a_{i} \mathrm{e}^{-c z_{i}^{4}}} \leq \frac{\sum_{i=1}^{N} a_{i} z_{i}^{p}}{\sum_{i=1}^{N} a_{i}}
$$

Proof. Let us set $f(c)=\frac{\sum_{i=1}^{N} a_{i} z_{i}^{p} \mathrm{e}^{-c z_{i}^{4}}}{\sum_{i=1}^{N} a_{i} \mathrm{e}^{-c z_{i}^{4}}}$. By Hölder's inequality, the derivative

$$
f^{\prime}(c)=\left(\frac{\sum_{i=1}^{N} a_{i} z_{i}^{p} \mathrm{e}^{-c z_{i}^{4}}}{\sum_{i=1}^{N} a_{i} \mathrm{e}^{-c z_{i}^{4}}} \frac{\sum_{i=1}^{N} a_{i} z_{i}^{4} \mathrm{e}^{-c z_{i}^{4}}}{\sum_{i=1}^{N} a_{i} \mathrm{e}^{-c z_{i}^{4}}}\right)-\frac{\sum_{i=1}^{N} a_{i} z_{i}^{p+4} \mathrm{e}^{-c z_{i}^{4}}}{\sum_{i=1}^{N} a_{i} \mathrm{e}^{-c z_{i}^{4}}}
$$

is non positive. Hence for any $c \geq 0, f(c) \leq f(0)=\frac{\sum_{i=1}^{N} a_{i} z_{i}^{p}}{\sum_{i=1}^{N} a_{i}}$.

Lemma 12. The sequence $\left(\eta_{n}(g)\right)_{1 \leq n \leq \nu}$ is bounded from below by a positive constant non depending on $\kappa$.

Proof. Since

$$
\eta_{n}(g)=\frac{\mathbb{E}\left(\exp \left(-\theta \delta t \sum_{k=1}^{n \kappa} X_{k \delta t}^{4}\right)\right)}{\mathbb{E}\left(\exp \left(-\theta \delta t \sum_{k=1}^{(n-1) \kappa} X_{k \delta t}^{4}\right)\right)} \leq 1
$$

the sequence $\left(\eta_{n}(g)\right)_{1 \leq n \leq \nu}$ is bounded from below by

$$
\prod_{n=1}^{\nu} \eta_{n}(g)=\mathbb{E}\left(\exp \left(-\theta \delta t \sum_{k=1}^{\nu \kappa} X_{k \delta t}^{4}\right)\right) .
$$

According to Lemma 2, this expectation converges to $\mathbb{E}\left(\exp \left(-\theta \int_{0}^{T} X_{s}^{4} \mathrm{~d} s\right)\right)>0$ when $\kappa$ tends to $+\infty$, which concludes the proof. 
We can now prove, as a corollary of Theorem 4 , the convergence of the approximation $\overline{E_{\mathrm{DMC}}^{N, \nu, \kappa}}(T)$ of $E_{\mathrm{DMC}}(T)$, defined by:

$$
\overline{E_{\mathrm{DMC}}^{N, \nu, \kappa}}(T)=\frac{3}{2} \omega+\frac{\theta}{N} \sum_{i=1}^{N}\left(X_{\nu+1,0}^{i}\right)^{4}
$$

\section{Corollary 13.}

$$
\mathbb{E}\left|E_{\mathrm{DMC}}(T)-\overline{E_{\mathrm{DMC}}^{N, \nu, \kappa}}(T)\right| \leq \frac{C}{\nu \kappa}+\frac{C_{\nu}}{\sqrt{N}}
$$

where the constant $C$ only depends on $T$ and the constant $C_{\nu}$ on $T$ and $\nu$.

Proof. By using the result of Theorem 4 and Cauchy Schwartz inequality, it is sufficient to prove the estimate $\mathbb{E}\left(E_{\mathrm{DMC}}^{N, \nu, \kappa}(T)-\overline{E_{\mathrm{DMC}}^{N, \nu, \kappa}}(T)\right)^{2} \leq \frac{C_{\nu}}{N}$. Let us denote in this proof $\xi_{\nu+1,0}^{i}=X_{\nu+1,0}^{i}$ for $1 \leq i \leq N$. We have:

$$
E_{\mathrm{DMC}}^{N, \nu, \kappa}(T)-\overline{E_{\mathrm{DMC}}^{N, \nu, \kappa}}(T)=\theta\left(\frac{\eta_{\nu}^{N}\left(g y_{\kappa}^{4}\right)}{\eta_{\nu}^{N}(g)}-\frac{1}{N} \sum_{i=1}^{N}\left(\xi_{\nu+1,0}^{i}\right)^{4}\right)=\theta\left(\mathbb{E}\left(\frac{1}{N} \sum_{i=1}^{N}\left(\xi_{\nu+1,0}^{i}\right)^{4} \mid \xi_{\nu}\right)-\frac{1}{N} \sum_{i=1}^{N}\left(\xi_{\nu+1,0}^{i}\right)^{4}\right)
$$

by using the fact that, for any function $f: \mathbb{R}_{+}^{*} \rightarrow \mathbb{R}_{+}$,

$$
\mathbb{E}\left(\frac{1}{N} \sum_{i=1}^{N} f\left(\xi_{\nu+1,0}^{i}\right) \mid \xi_{\nu}\right)=\frac{\eta_{\nu}^{N}\left(g(y) f\left(y_{\kappa}\right)\right)}{\eta_{\nu}^{N}(g(y))}
$$

which is obtained by a reasoning similar to the one made to prove (43). Now, using the same method as to obtain (44), one easily gets the estimate:

$$
\mathbb{E}\left(E_{\mathrm{DMC}}^{N, \nu, \kappa}(T)-\overline{E_{\mathrm{DMC}}^{N, \nu, \kappa}}(T)\right)^{2} \leq \frac{\theta^{2}}{N} \mathbb{E}\left(\frac{1}{N} \sum_{i=1}^{N}\left(\xi_{\nu+1,0}^{i}\right)^{8}\right)=\frac{\theta^{2}}{N} \mathbb{E}\left(\frac{\eta_{\nu}^{N}\left(g(y)\left(y_{\kappa}\right)^{8}\right)}{\eta_{\nu}^{N}(g(y))}\right)
$$

by using again (47). Lemma 9 completes the proof.

We end this section by proving that Proposition 6 also holds for the numerical scheme (17).

Proposition 14. Let us consider the Markov chain $\left(\bar{X}_{j \delta t}\right)_{0 \leq j \leq K}$ generated by the explicit scheme (17) and denote by $Q$ its transition kernel. We now define the measure $\eta_{n}$ by replacing $\left(X_{j \delta t}\right)_{0 \leq j \leq K}$ with $\left(\bar{X}_{j \delta t}\right)_{0 \leq j \leq K}$ in (31), and we define accordingly the evolution of the particle system: conditionally on $\xi_{n}$, the vectors $\left(X_{n+1,0}^{i}, X_{n+1, \delta t}^{i}, \ldots, X_{n+1, \kappa \delta t}^{i}\right)_{1 \leq i \leq N}$ are independent, with $\left(X_{n+1,0}^{i}\right)_{1 \leq i \leq N}$ distributed according to the selection algorithm (S1) (see (23)), (S2) (see (27)) or (S3) (see (29)), and $\left(X_{n+1, j \delta t}^{i}\right)_{0 \leq j \leq \kappa}$ a Markov chain with transition kernel $Q$. Then, we have:

$$
\mathbb{E}\left|\frac{\eta_{\nu}^{N}\left(g y_{\kappa}^{4}\right)}{\eta_{\nu}^{N}(g)}-\frac{\eta_{\nu}\left(g y_{\kappa}^{4}\right)}{\eta_{\nu}(g)}\right| \leq \frac{C_{\nu}}{\sqrt{N}}
$$

Proof. Looking carefully at the proof of Proposition 6 above, one remarks that (39) holds in this framework as soon as Lemma 12 holds, and the following property, which replaces Lemma 10, is satisfied:

$$
\exists C>0, \forall x \in \mathbb{R}_{+}, Q f(x) \leq \mathrm{e}^{C \delta t}(1+f(x))-1 \text { for } f(x) \equiv x^{4} \text { and } f(x) \equiv x^{8}
$$


Let us first prove (48). We have: $Q f(x)=\mathbb{E}\left(f\left(\bar{X}_{\delta t}^{x}\right)\right)$ where $\bar{X}_{\delta t}^{x}=\left((1-\omega \delta t)^{2} x^{2}+2 x W_{\delta t}+\frac{W_{\delta t}^{2}}{(1-\omega \delta t)^{2}}+2 \delta t\right)^{1 / 2}$. Now, for $q \in \mathbb{N}^{*}$,

$$
\left(\bar{X}_{\delta t}^{x}\right)^{2 q}=\sum_{j_{1}+j_{2}+j_{3}=q} \frac{q !}{j_{1} ! j_{2} ! j_{3} !}(1-\omega \delta t)^{2 j_{1}} 2^{j_{2}} x^{2 j_{1}+j_{2}} W_{\delta t}^{j_{2}}\left(\frac{W_{\delta t}^{2}}{(1-\omega \delta t)^{2}}+2 \delta t\right)^{j_{3}}
$$

where the indices $\left(j_{1}, j_{2}, j_{3}\right)$ are non negative integers. Remarking that the expectation of the terms with $j_{2}$ odd vanishes and then using Young's inequality, one deduces that for $\delta t \leq \frac{1}{2 \omega}$,

$$
\begin{aligned}
\mathbb{E}\left(\left(\bar{X}_{\delta t}^{x}\right)^{2 q}\right) & \leq(1-\omega \delta t)^{2 q} x^{2 q}+\mathbb{E}\left(\left(\frac{W_{\delta t}^{2}}{(1-\omega \delta t)^{2}}+2 \delta t\right)^{q}\right)+C_{q} \sum_{\substack{j_{1}+j_{2}+j_{3}=q \\
j_{1}<q, j_{2} \text { even } j_{3}<q}} x^{2\left(q-\frac{j_{2}+2 j_{3}}{2}\right)} \delta t^{\frac{j_{2}+2 j_{3}}{2}}, \\
& \leq x^{2 q}+C_{q} \delta t+C_{q} \sum_{\substack{j_{1}+j_{2}+j_{3}=q \\
j_{1}<q, j_{2} \text { even } j_{3}<q}}\left(x^{2 q} \delta t+\delta t^{1+q\left(1-\frac{2}{j_{2}+2 j_{3}}\right)}\right), \\
& \leq\left(1+C_{q} \delta t\right) x^{2 q}+C_{q} \delta t \leq \mathrm{e}^{C_{q} \delta t}\left(1+x^{2 q}\right)-1 .
\end{aligned}
$$

Let us now prove Lemma 12 for the scheme (17). As noticed in the proof of Lemma 12 above, it is sufficient to bound from below $\mathbb{E}\left(\exp \left(-\theta \delta t \sum_{k=1}^{\nu \kappa} \bar{X}_{k \delta t}^{4}\right)\right)$. By Jensen inequality, we have $\mathbb{E}\left(\exp \left(-\theta \delta t \sum_{k=1}^{\nu \kappa} \bar{X}_{k \delta t}^{4}\right)\right) \geq$ $\exp \left(-\theta \frac{T}{\nu \kappa} \sum_{k=1}^{\nu \kappa} \mathbb{E}\left(\bar{X}_{k \delta t}^{4}\right)\right)$. By using (49), it is easy to prove by induction that $\mathbb{E}\left(\bar{X}_{k \delta t}^{4}\right) \leq \mathrm{e}^{C_{2} k \delta t}\left(1+\mathbb{E}\left(\bar{X}_{0}^{4}\right)\right)-1$ and this concludes the proof of Lemma 12 in this framework.

In order to obtain a complete convergence result of the form (38) for the scheme (17), it remains to prove the complementary bound (19), that we have not obtained so far. However, we will check by numerical simulations that (38) still holds.

\section{Numerical RESUlts}

\subsection{Computation of a reference solution by a spectral method}

In this section, we would like to explain how we can obtain a very precise reference solution by using a partial differential equation approach to compute $E_{\mathrm{DMC}}(T)$ (see [4]).

\subsubsection{A partial differential equation approach to compute $E_{\mathrm{DMC}}(T)$}

Let us introduce the solution $\phi$ to the following partial differential equation:

$$
\left\{\begin{array}{l}
\frac{\partial \phi}{\partial t}=-H \phi,(t, x) \in \mathbb{R}_{+} \times \mathbb{R} \\
\phi(0, x)=\psi_{I}(x), x \in \mathbb{R}
\end{array}\right.
$$

where $H$ (resp. $\psi_{I}$ ) is defined by (10) (resp. (12)). Since $\psi_{I} \in \mathcal{H}$, it is a standard result that this problem admits a unique solution $\phi \in C^{0}\left(\mathbb{R}_{+}, \mathcal{H}\right) \cap C^{0}\left(\mathbb{R}_{+}^{*}, D_{\mathcal{H}}(H)\right) \cap C^{1}\left(\mathbb{R}_{+}^{*}, \mathcal{H}\right)$. The function $\phi$ is regular and odd, and therefore is such that $\phi(t, 0)=0$ for all $t \geq 0$. Therefore the function $\phi$ is also solution to the following partial differential equation:

$$
\left\{\begin{array}{l}
\frac{\partial \phi}{\partial t}=-H \phi,(t, x) \in \mathbb{R}_{+} \times \mathbb{R} \\
\phi(t, 0)=0, t \geq 0 \\
\phi(0, x)=\psi_{I}(x), x \in \mathbb{R} .
\end{array}\right.
$$


In [4], we have shown that since $\phi$ satisfies (51), we can express $E_{\mathrm{DMC}}(t)$ (defined by (2)) using the function $\phi$ (see Prop. 11 in [4]):

$$
E_{\mathrm{DMC}}(t)=\frac{\left\langle H \psi_{I}, \phi(t)\right\rangle}{\left\langle\psi_{I}, \phi(t)\right\rangle}
$$

Our reference solution $E_{\mathrm{DMC}}(T)$ will rely on formula (52) after discretization of (50) by a spectral method.

\subsubsection{Computation of the wave function $\phi$}

We will briefly present the spectral method developed to compute an approximation of $\phi$. We recall that the Hermite polynomials are defined by:

$$
\forall n \in \mathbb{N}, h_{n}(x)=(-1)^{n} \mathrm{e}^{x^{2}} \frac{\mathrm{d}^{n}}{\mathrm{~d} x^{n}}\left(\mathrm{e}^{-x^{2}}\right)
$$

We introduce the eigenfunctions of the operator $H_{0}$, normalized for the $L^{2}(\mathbb{R})$ norm associated with the eigenvalues $E_{n}=\omega(n+1 / 2)$ for $n \geq 0$,

$$
\varphi_{n}(x)=h_{n}(\sqrt{\omega} x) \exp \left(-\frac{1}{2} \omega x^{2}\right)\left(\frac{(\omega / \pi)^{1 / 4}}{\sqrt{2^{n} n !}}\right) .
$$

It is well known that the vector space spanned by the set of functions $\left\{\varphi_{2 k+1}\right\}_{k \geq 0}$ is dense in $\mathcal{V}_{0}=\{\varphi \in$ $\left.H^{1}(\mathbb{R}) \cap \mathcal{H} \mid x \varphi \in L^{2}\right\}$, which is the domain of the quadratic form associated with $\bar{H}_{0}$.

Let us now introduce the functional space $\mathcal{V}=\left\{\varphi \in H^{1}(\mathbb{R}) \cap \mathcal{H} \mid x^{2} \varphi \in L^{2}\right\}$, which is the domain of the quadratic form associated with $H$. The set of functions $\left\{\varphi_{2 k+1}\right\}_{k \geq 0}$ is also a basis of $\mathcal{V}$.

Let $\mathcal{V}_{n}=\operatorname{Span}\left(\varphi_{1}, \varphi_{3}, \ldots, \varphi_{2 n-1}\right)$. We use this approximation space to build the following Galerkin scheme for (50): find $\phi_{n} \in C^{0}\left(\mathbb{R}_{+}, \mathcal{V}_{n}\right)$ such that ${ }^{3} \phi_{n}(0, x)=\psi_{I}$, and $\forall \varphi \in \mathcal{V}_{n}$

$$
\left\langle\frac{\partial \phi_{n}(t)}{\partial t}, \varphi\right\rangle=-\left\langle H \phi_{n}(x, t), \varphi\right\rangle \text {. }
$$

We diagonalize the operator $H$ restricted to $\mathcal{V}_{n}$. We denote by $\left(\varphi_{0}^{n}, \varphi_{2}^{n}, \ldots, \varphi_{n-1}^{n}\right)$ the eigenfunctions and by $E_{0}^{n}, E_{2}^{n}, \ldots, E_{n-1}^{n}$ the associated eigenvalues. Because of the symmetry of $H$, it is easy to check that $\mathcal{V}_{n}$ can also be spanned by $\left(\varphi_{0}^{n}, \varphi_{2}^{n}, \ldots, \varphi_{n-1}^{n}\right)$ :

$$
\mathcal{V}_{n}=\operatorname{Span}\left(\varphi_{0}^{n}, \varphi_{2}^{n}, \ldots, \varphi_{n-1}^{n}\right)
$$

Since for $t \geq 0, \phi_{n}(t,.) \in \mathcal{V}_{n}$, there exists $u_{k}(t), k=0, \ldots, n-1$, such that

$$
\phi_{n}=\sum_{k=0}^{n-1} u_{k}(t) \varphi_{k}^{n}
$$

In view of (54) and (55), (53) is equivalent to the equations: $\forall i=0, \ldots, n-1$,

$$
\begin{aligned}
\sum_{k=0}^{n-1} \frac{\partial u_{k}(t)}{\partial t}\left\langle\varphi_{k}^{n}, \varphi_{i}^{n}\right\rangle & =-\left\langle H \sum_{k=0}^{n-1} u_{k}(t) \varphi_{k}^{n}, \varphi_{i}^{n}\right\rangle \\
& =-\sum_{k=0}^{n-1} E_{k}^{n} u_{k}(t)\left\langle\varphi_{k}^{n}, \varphi_{i}^{n}\right\rangle
\end{aligned}
$$

\footnotetext{
${ }^{3}$ Notice that $\psi_{I}=\varphi_{1} \in \mathcal{V}_{n}$.
} 
We deduce that $\forall k=0, \ldots, n-1$,

$$
\frac{\partial u_{k}(t)}{\partial t}=-E_{k}^{n} u_{k}(t)
$$

so that

$$
\phi_{n}(t, x)=\sum_{k=0}^{n-1} u_{k}(0) \exp \left(-E_{k}^{n} t\right) \varphi_{k}^{n}(x)
$$

where $u_{k}(0)=\left\langle\psi_{I}, \varphi_{k}^{n}\right\rangle$.

Remark 15. The eigenfunctions of $H$ are obtained by diagonalization of the matrix $A=\left(a_{i j}\right)_{i, j=0, \ldots, n-1}$ with $\forall i, j=0, \ldots, n-1$ :

$$
\begin{aligned}
a_{i j} & =\left\langle H \varphi_{2 i+1}, \varphi_{2 j+1}\right\rangle, \\
& =\left\langle H_{0} \varphi_{2 i+1}, \varphi_{2 j+1}\right\rangle+\theta\left\langle x^{4} \varphi_{2 i+1}, \varphi_{2 j+1}\right\rangle, \\
& =\delta_{i j} \omega\left(2 i+\frac{3}{2}\right)+\theta\left\langle x^{4} \varphi_{2 i+1}, \varphi_{2 j+1}\right\rangle .
\end{aligned}
$$

We can use the $n$-point Gauss-Hermite formula to deal with the integration of the second term on the right-hand side. We recall that this method provides an exact result for $\int_{-\infty}^{+\infty} p(x) \exp \left(-x^{2}\right) \mathrm{d} x$ as long as $p$ is a polynomial of degree $2 n-1$ or less.

\subsubsection{Approximation of $E_{\mathrm{DMC}}(T)$}

We now use formula (52) to approximate $E_{\mathrm{DMC}}(T)$. By an elementary calculation, we obtain the following approximation:

$$
E_{\mathrm{DMC}}(T) \simeq \frac{E_{0}^{n}+\sum_{i=1}^{n-1} \frac{u_{i}(0)\left\langle\varphi_{i}^{n}, \varphi_{1}\right\rangle}{u_{0}(0)\left\langle\varphi_{1}^{n}, \varphi_{1}\right\rangle} E_{i}^{n} \exp \left(-\left(E_{i}^{n}-E_{0}^{n}\right) T\right)}{1+\sum_{i=1}^{n-1} \frac{u_{i}(0)\left\langle\varphi_{i}^{n}, \varphi_{1}\right\rangle}{u_{0}(0)\left\langle\varphi_{1}^{n}, \varphi_{1}\right\rangle} \exp \left(-\left(E_{i}^{n}-E_{0}^{n}\right) T\right)} .
$$

In our test cases, we have observed that $n=40$ is enough to reach convergence.

Notice that for a given $n$, the convergence in time to the lowest eigenvalue $E_{0}^{n}$ is exponentially fast, with an exponent equal to the spectral gap $E_{1}^{n}-E_{0}^{n}$.

\subsection{Numerical results of Monte Carlo simulations}

In this section, we perform various numerical experiments to validate our theoretical results, and to explore some features of DMC computation. In particular, we propose in Section 2.2.2 an empirical method to determine the optimal number of reconfigurations. In all the computations, the final time is $T=5$, which appears to be sufficiently large for the convergence $t \rightarrow \infty$ to be achieved with enough accuracy.

\subsubsection{Error and variance as a function of the numerical parameters}

We represent on Figure 2, the expectation $e$ and the variance $v$ of the error: $\left|E_{\mathrm{DMC}}^{N, \nu, T /(\nu \delta t)}(T)-E_{\mathrm{DMC}}(T)\right|$ as a function of the number of walkers $N$, the time step $\delta t$ and the number of reconfigurations $\nu-1$, where $E_{\mathrm{DMC}}(T)$ is approximated using $(57)$ and $E_{\mathrm{DMC}}^{N, \nu, T /(\nu \delta t)}(T)$ is defined by (37). The multinomial resampling method (which is (S1) with $\epsilon_{n}=0$ ) was used.

The top figures represent the expectation of the error and its variance according to the number of walkers. To compute these quantities, we perform 2000 independent realizations, with the number of reconfigurations $\nu-1=50$, a small time step $\delta t=5 \times 10^{-3}$ and $\theta=0.5$. The simulations confirm the theoretical result: the error decreases as $C / \sqrt{N}$.

The effect of the time step is shown on the two figures in the center. The numerical parameters are: a large number of particles $N=5000$, number of configurations $\nu-1=30, \theta=2$ and 300 independent realizations. We can see on the figure on the left that the error decreases linearly as the time step decreases. We also remark that the error is smaller with the approximate scheme (17) than when using the exact simulation of 

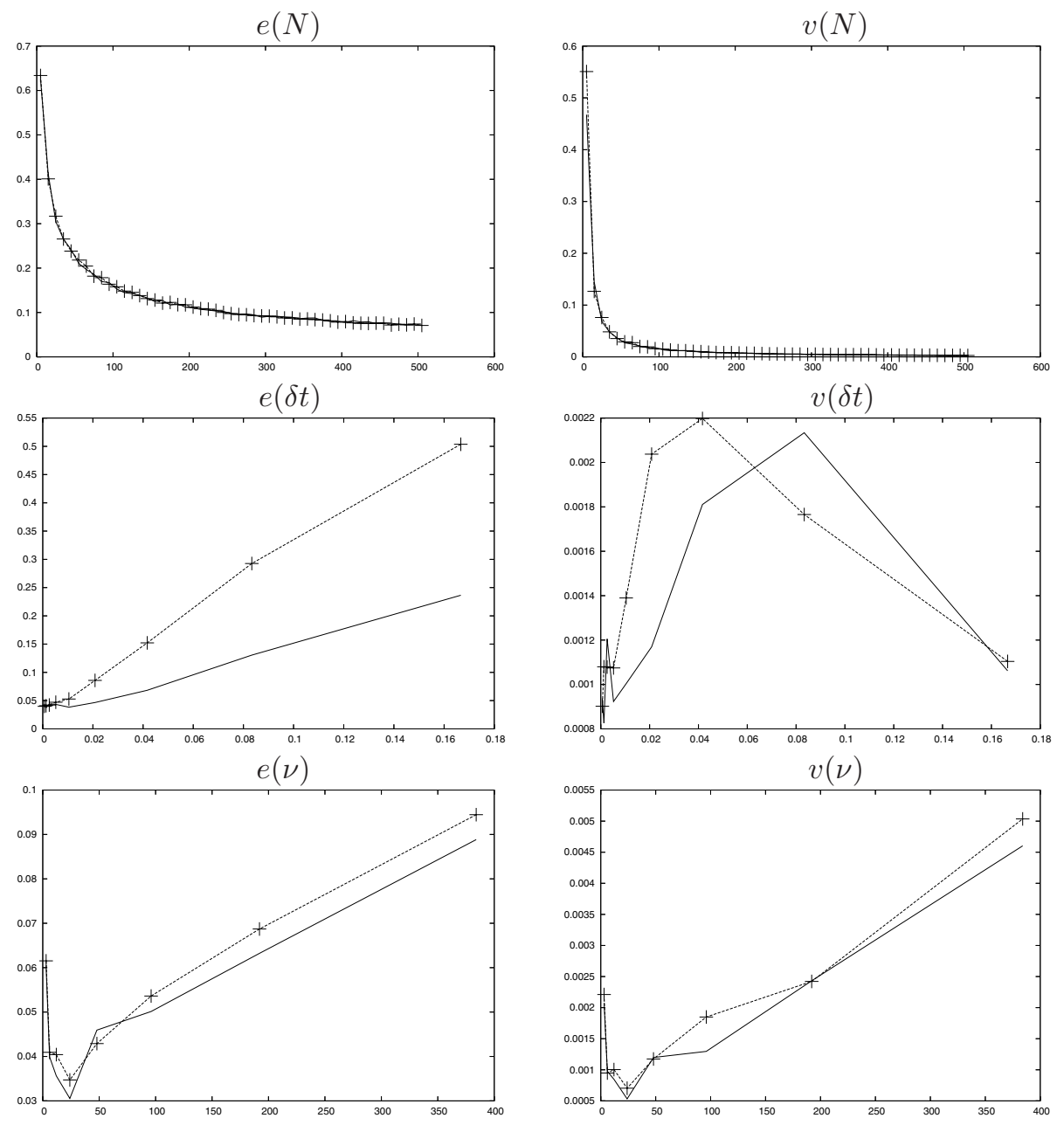

FIGURE 2. Expectation and variance of the error when (15) is discretized according to the method described in Appendix (dotted curve) and according to the scheme (17) (solid curve).

the SDE (15) proposed in the Appendix. This rather amazing result can be interpreted as follows. When using the exact simulation of the SDE, there is only one source of error related to the time discretization, namely the approximation of the integral in the exponential factor in (2). When using the scheme (17), we add a weak error term which seems to partly compensate the previous one.

The last figures represent the effect of the number of reconfiguration steps. The numerical parameters are: time step $\delta t=5 \times 10^{-3}$, number of particles $N=5000, \theta=2$ and 300 independent realizations. The curve representing the variation of the error according to the number of reconfigurations has the shape of a basin. We deduce that on the one hand a small number of reconfigurations has the disadvantage that walkers with increasingly differing weights are kept. On the other hand a large number of reconfigurations introduces much noise. An optimal number of reconfiguration seems to lie between 20 and 50.

\subsubsection{Optimal number of reconfigurations}

In Figure 3, we check that the optimal number of reconfigurations in terms of the variance $\tilde{v}$ of $E_{\mathrm{DMC}}^{N, \nu, T /(\nu \delta t)}(T)$ (and not of the error as in Sect. 2.2.1) is also obtained for a number of reconfiguration which seems to lie between 


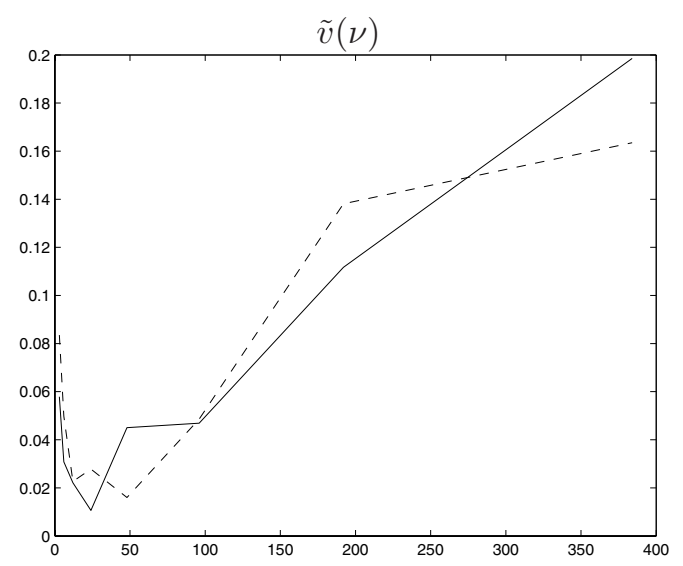

FIGURE 3. Variance of $E_{\mathrm{DMC}}^{N, \nu, T /(\nu \delta t)}(T)$ in function of the number of reconfigurations when (15) is discretized according to the method described in Appendix (solid curve) and according to the scheme (17) (dashed curve).

20 and 50 (using again the multinomial resampling method). The numerical parameters are those considered for the figures below in Figure 2: time step $\delta t=5 \times 10^{-3}$, number of particles $N=5000, \theta=2$ and 300 independent realizations. We have not studied how the optimal number of reconfigurations varies according to the other numerical parameters.

We have investigated a practical method to estimate numerically the optimal number of reconfigurations. In Figure 4 we represent the variance of $E_{\mathrm{DMC}}^{N, 1, t / \delta t}(t)$ according to time $t$, without any reconfiguration step (which corresponds to $\nu=1$ ). The other numerical parameters are again those considered for the figures below in Figure 2. We observe that the variance is minimal at $t^{*} \approx 0.25$. We remark that $\nu=T / t^{*}=20$ is close to the optimal number of reconfigurations obtained on the previous figures. We have checked this empirical result for various sets of the parameters. It seems that the optimal number of reconfigurations is related to $T / t^{*}$ where $t^{*}$ minimizes the variance of $E_{\mathrm{DMC}}^{N, 1, t / \delta t}(t)$. Since $\nu=1$, no selection step occurs and the particles are thus independent. According to the multidimensional central limit theorem, the variance of $E_{\mathrm{DMC}}^{N, 1, t / \delta t}(t)$ can be approximated by

$$
\frac{1}{N}\left(\frac{\operatorname{Var}\left(Y_{t}\right)}{\left(\mathbb{E}\left(Z_{t}\right)\right)^{2}}-2 \mathbb{E}\left(Y_{t}\right) \frac{\operatorname{Covar}\left(Y_{t}, Z_{t}\right)}{\left(\mathbb{E}\left(Z_{t}\right)\right)^{3}}+\left(\mathbb{E}\left(Y_{t}\right)\right)^{2} \frac{\operatorname{Var}\left(Z_{t}\right)}{\left(\mathbb{E}\left(Z_{t}\right)\right)^{4}}\right)
$$

where

$$
Y_{t}=E_{L}\left(X_{t}\right) \exp \left(-\delta t \sum_{k=1}^{t / \delta t} E_{L}\left(X_{k \delta t}\right)\right)
$$

and

$$
Z_{t}=\exp \left(-\delta t \sum_{k=1}^{t / \delta t} E_{L}\left(X_{k \delta t}\right)\right)
$$

Therefore, the optimal number of reconfiguration steps could be estimated by this method, through a precomputation over a few independent trajectories. 


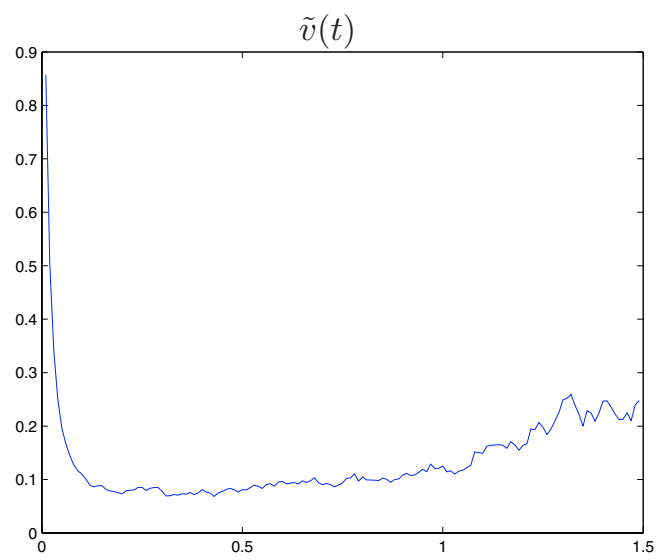

FiguRE 4. Variance of $E_{\mathrm{DMC}}^{N, 1, t / \delta t}(t)$ as a function of time $t$.
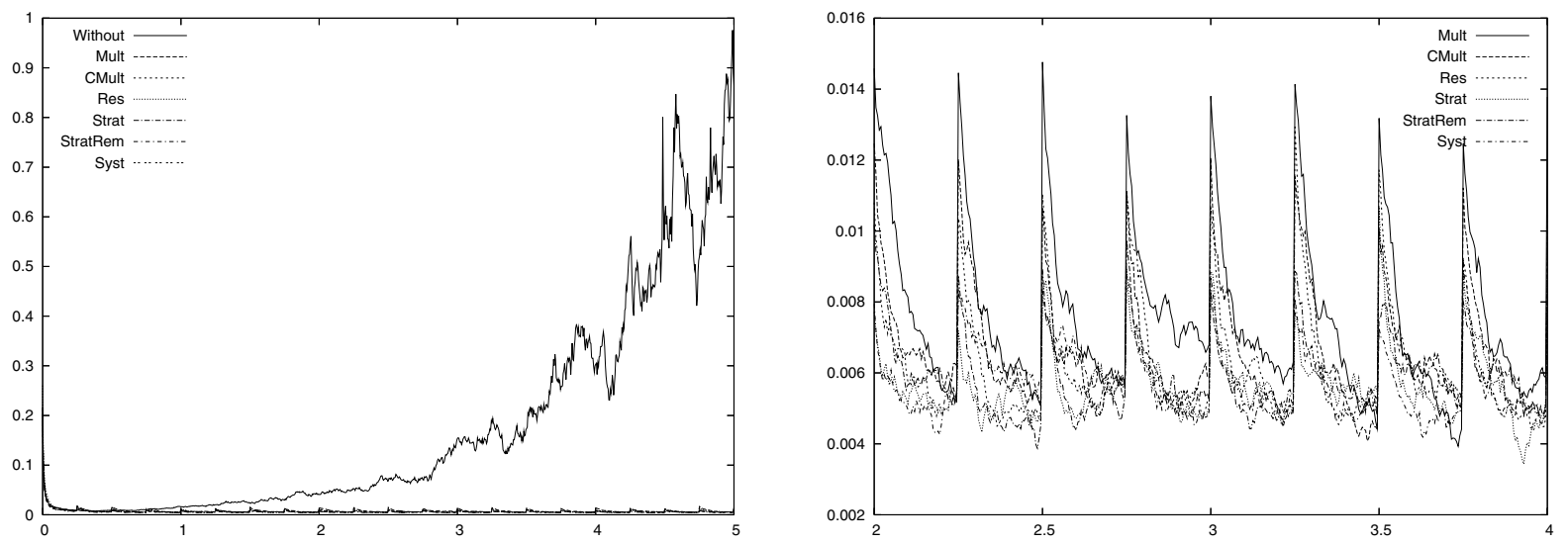

FIGURE 5. Variance of $E_{\mathrm{DMC}}^{N, \nu, T /(\nu \delta t)}(t)$ as a function of time $t$, for various resampling algorithms: Without $=$ without resampling, Mult $=$ multinomial resampling, CMult $=$ correlated multinomial resampling, Res $=$ residual resampling, Strat $=$ stratified resampling, StratRem $=$ stratified remainder resampling, Syst $=$ systematic resampling.

\subsubsection{Comparison of the resampling algorithms}

We finally compare various resampling algorithms in Figure 5 , where the variance of $E_{\mathrm{DMC}}^{N, \nu, T /(\nu \delta t)}(t)$ as a function of time is represented. The numerical parameters are: $N=1000, \delta t=5 \times 10^{-3}, \nu-1=20, \theta=2$ and 200 independent realizations.

We first observe on the figure on the left that without any resampling, the variance of the results explodes with increasing time. This shows the necessity to use resampling algorithms. We compare the following resampling algorithms: multinomial resampling (which is (S1) with $\epsilon_{n}=0$ ), correlated multinomial resampling (which is (S1) with $\epsilon_{n}=1 / \max _{1 \leq i \leq N} g\left(\xi_{n}^{i}\right)$ ), residual resampling (which is (S2)), stratified resampling (which is (S3)), stratified remainder resampling (which combines residual and stratified resampling, see Rem. 8) and systematic resampling (which corresponds to stratified resampling with $U_{n}^{1}=\ldots=U_{n}^{N}=U_{n}$, see the Introduction). We observe that, as expected, when more correlation is introduced, the variance due to the resampling is reduced. 
The multinomial resampling method is generally the worse, while the best resampling methods seem to be systematic resampling or stratified remainder resampling.

\section{Conclusion}

In this paper, we have proved on a simple example convergence of numerical implementations of the DMC method with a fixed number of walkers. The theoretical rates of convergence are confirmed by numerical experiments and are likely to hold in more general situations. We have also checked numerically the existence of an optimal number of reconfiguration steps. Various resampling algorithms have been considered, both theoretically and numerically. For future work, we plan to investigate criteria devoted to the choice of the number of reconfiguration steps. One interesting direction is the use of automatic criteria based on a measure of the discrepancy between the weights carried by the walkers to decide when to perform a reconfiguration step.

\section{Appendix : Simulation of the Stochastic Differential Equation (15)}

In this appendix, we show that it is possible to simulate exactly in law the $(K+1)$-plet $\left(X_{0}, X_{\delta t}, \ldots, X_{K \delta t}\right)$, where $X_{t}$ is defined by $(15)$. Let $(G, U)$ denote a couple of independent random variables with $G$ normal and $U$ uniformly distributed on the interval $[0,1]$.

Simulation of the increment $X_{t}-X_{s}$, for $t \geq s$.

The square $R_{t}$ of the norm of a 3-dimensional Brownian motion $\boldsymbol{W}_{t}=\left(\boldsymbol{W}_{t}^{1}, \boldsymbol{W}_{t}^{2}, \boldsymbol{W}_{t}^{3}\right)$ solves $\mathrm{d} R_{t}=3 \mathrm{~d} t+$ $2 \sqrt{R_{t}} \mathrm{~d} B_{t}$ where $B_{t}=\int_{0}^{t} \frac{\boldsymbol{W}_{s} \cdot \mathrm{d} \boldsymbol{W}_{s}}{\left\|\boldsymbol{W}_{s}\right\|}$ is a one-dimensional Brownian motion. Hence $\rho_{t}=\frac{R_{t}}{1+2 \omega t}$ solves

$$
\mathrm{d} \rho_{t}=\left(3-2 \omega \rho_{t}\right) \frac{\mathrm{d} t}{1+2 \omega t}+2 \sqrt{\rho_{t}} \frac{\mathrm{d} B_{t}}{\sqrt{1+2 \omega t}}
$$

It is easy to check that $\left(\int_{0}^{\frac{1}{2 \omega}\left(\mathrm{e}^{2 \omega t}-1\right)} \frac{\mathrm{d} B_{s}}{\sqrt{1+2 \omega s}}\right)_{t}$ is a Brownian motion. Hence, performing a time-change in (58), one obtains that $\rho_{\frac{1}{2 \omega}\left(\mathrm{e}^{2 \omega t}-1\right)}=\mathrm{e}^{-2 \omega t} R_{\frac{1}{2 \omega}\left(\mathrm{e}^{2 \omega t}-1\right)}$ is a weak solution of the equation $\mathrm{d} Y_{t}=\left(3-2 \omega Y_{t}\right) \mathrm{d} t+$ $2 \sqrt{Y_{t}} \mathrm{~d} W_{t}$ satisfied by $Y_{t}=X_{t}^{2}$. Therefore $\mathrm{e}^{-\omega t} \sqrt{R_{\frac{1}{2 \omega}\left(\mathrm{e}^{2 \omega t}-1\right)}}$ is a weak solution of (15).

For $v \geq u, R_{v}$ has the same distribution as $\left(\sqrt{R_{u}}+\boldsymbol{W}_{v}^{1}-\boldsymbol{W}_{u}^{1}\right)^{2}+\left(\boldsymbol{W}_{v}^{2}-\boldsymbol{W}_{u}^{2}\right)^{2}+\left(\boldsymbol{W}_{v}^{3}-\boldsymbol{W}_{u}^{3}\right)^{2}$, and therefore as $\left(\sqrt{R_{u}}+G \sqrt{v-u}\right)^{2}-2(v-u) \log (U)$ with $(G, U)$ independent from $R_{u}$. Hence for $t \geq s, X_{t}$ has the same distribution as

$$
\begin{aligned}
& \left(\mathrm{e}^{-2 \omega t}\left(\left(\mathrm{e}^{\omega s} X_{s}+\frac{G}{\sqrt{2 \omega}}\left(\mathrm{e}^{2 \omega t}-\mathrm{e}^{2 \omega s}\right)^{1 / 2}\right)^{2}-2 \frac{1}{2 \omega}\left(\mathrm{e}^{2 \omega t}-\mathrm{e}^{2 \omega s}\right) \log (U)\right)\right)^{1 / 2} \\
& =\left(\left(\mathrm{e}^{-\omega(t-s)} X_{s}+\frac{G}{\sqrt{2 \omega}}\left(1-\mathrm{e}^{-2 \omega(t-s)}\right)^{1 / 2}\right)^{2}-\frac{1}{\omega}\left(1-\mathrm{e}^{-2 \omega(t-s)}\right) \log (U)\right)^{1 / 2}
\end{aligned}
$$

where the couple $(G, U)$ is independent from $X_{s}$.

Simulation of $X_{0}$ with distribution $2 \psi_{I}^{2}(x) 1_{\{x>0\}} \mathrm{d} x$.

The random variable $\frac{1}{\sqrt{2 \omega}}\left(G^{2}-2 \log (U)\right)^{1 / 2}$ is distributed according to the invariant measure $2 \psi_{I}^{2}(x) 1_{\{x>0\}} \mathrm{d} x$, as suggested by letting the time increment $t-s$ tend to $+\infty$ in the previous simulation. Indeed, $G^{2}-2 \log (U)$ is a Gamma random variable with density $\frac{1}{2^{3 / 2} \Gamma(3 / 2)} 1_{\{z>0\}} \sqrt{z} \mathrm{e}^{-z / 2}$. And one deduces the density of $\frac{1}{\sqrt{2 \omega}}\left(G^{2}-2 \log (U)\right)^{1 / 2}$ by an easy change of variables. 


\section{REFERENCES}

[1] A. Alfonsi, On the discretization schemes for the CIR (and Bessel squared) processes. Monte Carlo Methods Appl. 11 (2005) 355-384.

[2] R. Assaraf, M. Caffarel and A. Khelif, Diffusion Monte Carlo with a fixed number of walkers. Phys. Rev. E 61 (2000) $4566-4575$.

[3] E. Cancès, M. Defranceschi, W. Kutzelnigg, C. Le Bris and Y. Maday, Computational Quantum Chemistry: a Primer, in Handbook of Numerical Analysis, Special volume, Computational Chemistry, volume X, Ph.G. Ciarlet and C. Le Bris Eds., North-Holland (2003) 3-270.

[4] E. Cancès, B. Jourdain and T. Lelièvre, Quantum Monte Carlo simulations of fermions. A mathematical analysis of the fixed-node approximation. Math. Mod. Methods Appl. Sci. 16 (2006) 1403-1440.

[5] O. Cappé, R. Douc and E. Moulines, Comparison of Resampling Schemes for Particle Filtering, in 4th International Symposium on Image and Signal Processing and Analysis (ISPA), Zagreb, Croatia (2005).

[6] N. Chopin, Central limit theorem for sequential Monte Carlo methods and its application to Bayesian inference. Ann. Statist. 32 (2004) 2385-2411.

[7] P. Del Moral, Feynman-Kac Formulae: Genealogical and Interacting Particle Systems with Applications. Springer-Verlag (2004).

[8] P. Del Moral and A. Doucet, Particle motions in absorbing medium with hard and soft obstacles. Stochastic Anal. Appl. 22 (2004) 1175-1207.

[9] P. Del Moral and L. Miclo, Branching and Interacting Particle Systems. Approximation of Feynman-Kac Formulae with Applications to Non-Linear Filtering, in Séminaire de Probabilités XXXIV, Lecture Notes in Mathematics 1729, SpringerVerlag (2000) 1-145.

[10] P. Del Moral and L. Miclo, Particle approximations of Lyapunov exponents connected to Schrödinger operators and FeynmanKac semigroups. ESAIM: PS 7 (2003) 171-208.

[11] P. Glasserman, Monte Carlo methods in financial engineering. Springer-Verlag (2004).

[12] J.H. Hetherington, Observations on the statistical iteration of matrices. Phys. Rev. A 30 (1984) 2713-2719.

[13] P.J. Reynolds, D.M. Ceperley, B.J. Alder and W.A. Lester, Fixed-node quantum Monte Carlo for molecules. J. Chem. Phys. 77 (1982) 5593-5603.

[14] M. Rousset, On the control of an interacting particle approximation of Schrödinger groundstates. SIAM J. Math. Anal. 38 (2006) 824-844.

[15] S. Sorella, Green Function Monte Carlo with Stochastic Reconfiguration. Phys. Rev. Lett. 80 (1998) $4558-4561$.

[16] D. Talay and L. Tubaro, Expansion of the global error for numerical schemes solving stochastic differential equations. Stochastic Anal. Appl. 8 (1990) 94-120.

[17] C.J. Umrigar, M.P. Nightingale and K.J. Runge, A Diffusion Monte Carlo algorithm with very small time-step errors. J. Chem. Phys. 99 (1993) 2865-2890. 